\title{
Prospects of Searches for Neutral, Long-Lived Particles which Decay to Photons using Timing at CDF
}

\author{
David Tobach 7 and Peter Wagneli \\ Texas A $6 M$ University, College Station, Texas 77843-4242
}

\begin{abstract}
We present the prospects of searches for neutral, long-lived particles which decay to photons using their time of arrival measured with a newly installed timing system on the electromagnetic calorimeter (EMTiming) of the Collider Detector at Fermilab (CDF). A Monte Carlo simulation shows that EMTiming can provide separation between decay photons from particles with both a long lifetime and a low boost, and prompt photons from Standard Model backgrounds. Using a gauge mediated supersymmetry breaking (GMSB) $\tilde{\chi}_{1}^{0} \rightarrow \gamma \tilde{G}$ model we estimate a quasi-modelindependent sensitivity using only direct neutralino pair production, and also estimate the expected $95 \%$ confidence level exclusion regions for all superpartner production as a function of the neutralino mass and lifetime. We find that a combination of single photon and diphoton analyses should allow the Tevatron in run II to easily extend the exclusion regions from LEP II at high neutralino masses and lifetimes, and cover much, if not all, of the theoretically favored $m_{\tilde{G}}<1 \mathrm{keV} / c^{2}$ parameter space for neutralino masses less than $150 \mathrm{GeV} / c^{2}$.
\end{abstract}

\section{INTRODUCTION}

The electromagnetic (EM) calorimeter at the Collider Detector at Fermilab (CDF) [1] has recently been equipped with a new nanosecond-resolution timing system, EMTiming [2], to measure the arrival time of energy deposited (e.g. from photons). While it was initially designed to reject cosmics and accelerator backgrounds [3], we investigate the possibility of using it to search for neutral particles [4] with a lifetime of the order of a nanosecond which decay in flight to photons. An example of a theory which would produce these particles is the gauge mediated supersymmetry breaking (GMSB) model [5] with a neutralino, $\tilde{\chi}_{1}^{0}$, as the next-to-lightest supersymmetric particle (NLSP) and a light gravitino, $\tilde{G}$, as the LSP. In this scenario the neutralino decays preferably $(\sim 100 \%)$ as $\tilde{\chi}_{1}^{0} \rightarrow \gamma \tilde{G}$ with a macroscopic lifetime for much of the GMSB parameter space.

We begin with a study of the properties of events where timing can be used to separate between decay photons from long-lived particles, and photons produced promptly at the collision. A suitable variable to describe this distinction is the measured difference between the time after which the photon arrives at the face of the detector, and the time a prompt photon would virtually need to reach the same final position. This time difference for a prompt photon, from Standard Model (SM) sources, is exactly 0 but is always greater than 0 for photons from delayed decays, as in GMSB/Supersymmetry, if we neglect the measurement resolution. We call this difference $\Delta s$ :

$$
\Delta s \equiv\left(t_{f}-t_{i}\right)-\frac{\left|\vec{x}_{f}-\vec{x}_{i}\right|}{c}
$$

\footnotetext{
*Electronic address: toback@fnal.gov

†Electronic address: wagnp@fnal.gov
}

where $t_{f}-t_{i}$ is the time between the collision and the arrival time of the photon at the face of the detector, and $\left|\vec{x}_{f}-\vec{x}_{i}\right|$ is the distance between the final position of the photon and the collision point. The situation is visualized in Fig. [1 All four variables can be measured by the CDF detector [6] and give a system resolution of $\sigma_{\text {EMTiming }} \sim 1.0 \mathrm{~ns}[$ [7].

An important note in our analysis is that photons from long-lived particles will usually not arrive at the face of the EM calorimeter at the usual 90 degree incident angle. This could have serious implications for photon identification. For the purposes of this study we assume that this issue can be addressed without significant changes to the identification efficiency. We further assume that the additional handles such as EMTiming and timing in the hadronic calorimeters provide the necessary robustness needed to convince ourselves that photons which might not pass ordinary selection requirements are indeed from our signal source as opposed to sources which could produce fake photons and missing transverse energy, $\mathbb{E}_{T}$, like cosmics.

We estimate our sensitivity to two different types of new particle production using GMSB models. As a quasimodel-independent sensitivity estimate to generic longlived particles we simulate direct neutralino pair production and decay, and examine the dependency as a function of both neutralino mass, $m_{\tilde{\chi}}$, and lifetime, $\tau_{\tilde{\chi}}$. For a "full" GMSB model sensitivity, which means including all relevant GMSB subprocesses such that the neutralinos are part of cascades from gauginos and squarks, we allow all SUSY particle production and decay, and again vary the mass and lifetime variables. To choose analysis final states for both we consider three issues: 1) with neutralino lifetimes longer than a nanosecond it is possible that one or both of the neutralinos leave the detector before they decay, 2) with gravitinos or the neutralino leaving the detector $\mathbb{E}_{T}$ should also help separate signal from SM backgrounds and 3) to ensure that our predictions are as reliable as possible we want to use the data 


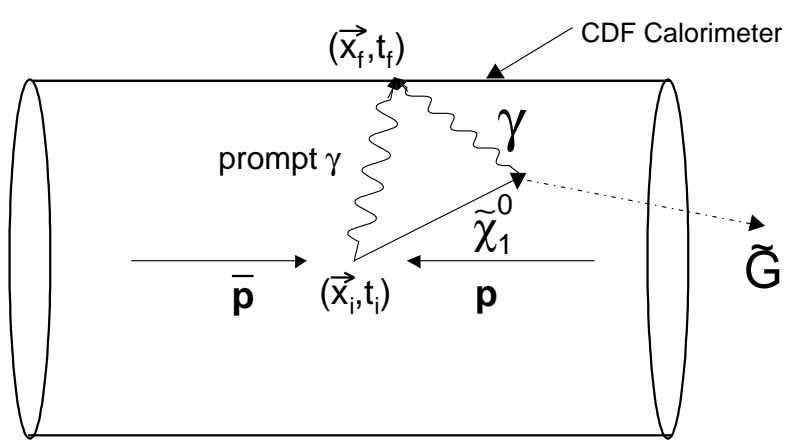

Figure 1: A schematic diagram of a long-lived neutralino decaying to a photon and a gravitino in the CDF detector. The neutralino emanates from the collision at $\left(\vec{x}_{i}, t_{i}\right)$ and after a time $\tau$ it decays. While the gravitino leaves the detector the photon travels to the detector wall and deposits energy in the EM calorimeter where its final location $\vec{x}_{f}$ and arrival time $t_{f}$ can be measured. A prompt photon would travel directly from $\vec{x}_{i}$ to $\vec{x}_{f}$ which can both be measured. The difference between the actual time the neutralino/photon needs, $\Delta t=t_{f}-t_{i}$, and the time a prompt photon would need, $\frac{\left|\vec{x}_{f}-\vec{x}_{i}\right|}{c}$, is defined as $\Delta s$. The SM typically produces prompt photons which have $\Delta s=0 \mathrm{~ns}$, whereas photons from delayed decays from SUSY have $\Delta s>0 \mathrm{~ns}$, assuming a perfect measurement.

selection requirements and background predictions from previously published papers by CDF [1] and D $\varnothing[8]$. In the 1992-1995 collider run (run I) of the Tevatron three types of analyses match these criteria: CDF and DØ results in $\gamma \gamma+E_{T}[3,9]$, exclusive $\gamma+\mathscr{E}_{T}\left(\gamma+\mathbb{E}_{T}+0\right.$ jets $)$ from $\mathrm{CDF}[10]$ and $\gamma+\mathscr{E}_{T}+$ jets from D $\varnothing$ [11]. Since there are no jets at the parton level in direct neutralino pair production, in this case we consider analyses with final states $\gamma \gamma+\mathscr{E}_{T}$ and $\gamma+\mathscr{E}_{T}+0$ jets. To estimate the sensitivity for full GMSB neutralino production we consider both $\gamma \gamma+\mathscr{E}_{T}$ and $\gamma+\mathscr{E}_{T}+$ jets analyses.

For both direct neutralino pair production and full GMSB production we quantify the sensitivity for $2 \mathrm{fb}^{-1}$ in run II using the expected $95 \%$ confidence level (C.L.) cross section upper limits. Results for both with and without the EMTiming system, using kinematics cuts only, illustrate the contribution to the final sensitivity from kinematic and timing information considerations [12]. Finally, we compare the final mass and lifetime exclusion regions for a GMSB scenario to direct and indirect searches from the ALEPH experiment 13] at LEP II and the theoretically favored parameter space from cosmological model restrictions of $m_{\tilde{G}}<1 \mathrm{keV} / c^{2}$ [14].

\section{KINEMATIC PROPERTIES OF EVENTS WITH LONG-LIVED PARTICLES WHICH DECAY TO PHOTONS}

While the final sensitivity studies use both a full physics generation and a detector simulation of the geometry and timing resolution, we begin with a study of the kinematic properties of events which yield large $\Delta s$ measurements using a "toy Monte Carlo." For now the CDF detector is assumed to be a cylinder, with length $3.5 \mathrm{~m}$ and radius $1.7 \mathrm{~m}$, instrumented with time and position detectors of perfect resolution for both the collision point and where the photon hits the face of the detector. Neutral particles, which we will refer to as neutralinos, are simulated as emanating isotropically from the center of the detector and emit a photon isotropically after a lifetime $\tau_{\tilde{\chi}}$ in their rest frame. For a promptly decaying photon the minimum time corresponds to the nearest distance to the detector face: $\frac{1.7 \mathrm{~m}}{c}=5.6 \mathrm{~ns}$; the maximum to the largest distance $\sqrt{(1.7 \mathrm{~m})^{2}+\left(\frac{3.5 \mathrm{~m}}{2}\right)^{2}} / c=8.1 \mathrm{~ns}$. For pedagogic reasons, neutralinos are simulated with a flat momentum and lifetime distribution, i.e. independently any lifetime and momentum have equal probability. We note that to be conservative here, as later in the paper, only those neutralinos that decay before the face of the detector are considered to have produced a photon.

Figure 2 shows the measured $\Delta s$ versus the event lifetime of the $\tilde{\chi}_{1}^{0}$ in the lab frame, $\tau_{\text {evt,L}}$. For $\Delta s \gtrsim 10 \mathrm{~ns}$ there is a roughly linear relation between $\Delta s$ and $\tau_{\text {evt,L }}$. For a fixed $\tau_{\text {evt }, \mathrm{L}}$ the maximum $\Delta s$ (upper bound) occurs when the neutralino travels to the farthest corner of the detector and then emits a photon backward to the opposite corner. Analogously we get a minimum $\Delta s$ (lower bound) if the neutralino travels with high momentum to the nearest part of the detector and emits a photon forward. The latter would look like a usual prompt photon event except for the difference in velocity between the neutralino and the photon. If the event lifetime is greater than the maximum time a prompt photon would need to travel to the detector then $\Delta s$ is restricted from below and $\Delta s>0$ ns (given that the neutralino decays inside the detector). Thus, the spread mainly comes from detector geometry but with the neutralino momentum also contributing to the width.

Figure 3 shows $\Delta s$ versus the neutralino boost for the lifetime slice $8.5 \mathrm{~ns} \leq \tau_{\text {evt, } \mathrm{L}} \leq 9.0 \mathrm{~ns}$. A low boost (between 1.0 and 1.5) allows large $\Delta s$ since neutralinos can have a larger lifetime without leaving the detector. Neutralinos with high boost are more likely to leave the detector, and even if they do not and their photon is detected, it has low $\Delta s(0 \mathrm{~ns} \lesssim \Delta s \lesssim 2 \mathrm{~ns})$. Thus, events with large $\Delta s$ are produced by neutralinos with long lifetimes and low boost.

Next we consider the efficiency for neutralinos to remain in the detector and/or produce a photon with large $\Delta s$. Figure 4 shows the efficiency, the fraction of all generated events that produce photons which pass a given 


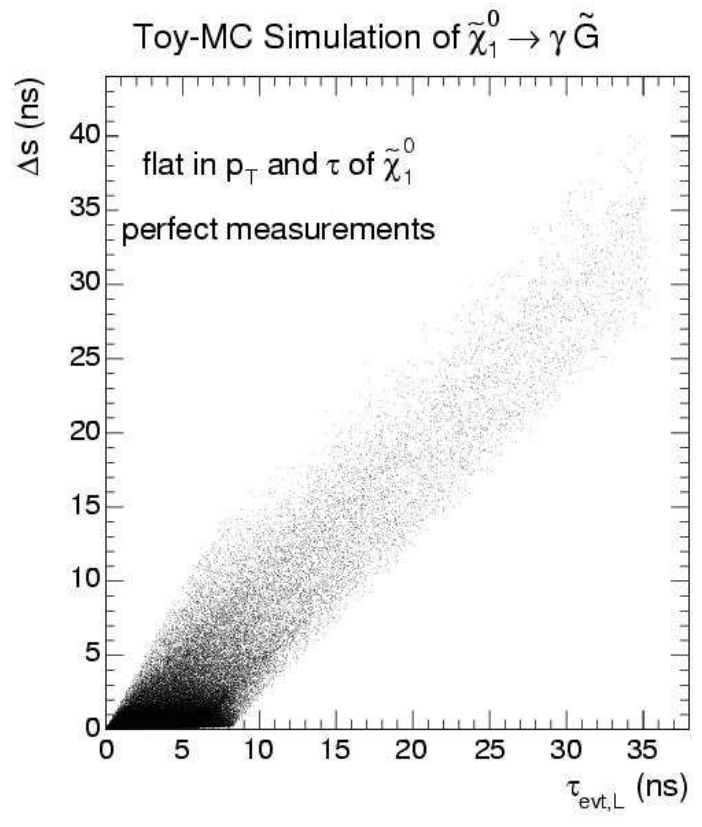

Figure 2: The $\Delta s$ distribution as a function of the event lifetime in the neutralino lab frame for a toy Monte Carlo simulation. In general, $\Delta s$ is proportional to $\tau_{\text {evt,L. At large }}$ $\tau_{\text {evt,L }}$ most of the neutralinos leave the detector and are not shown here. The spread perpendicular to $\Delta s \sim \tau_{\text {evt,L }}$ originates in variations of the neutralino momentum as well as in variations in the travel time of the photon due to detector geometry. Essentially, events with large $\Delta s$ require a neutralino with a long lifetime.

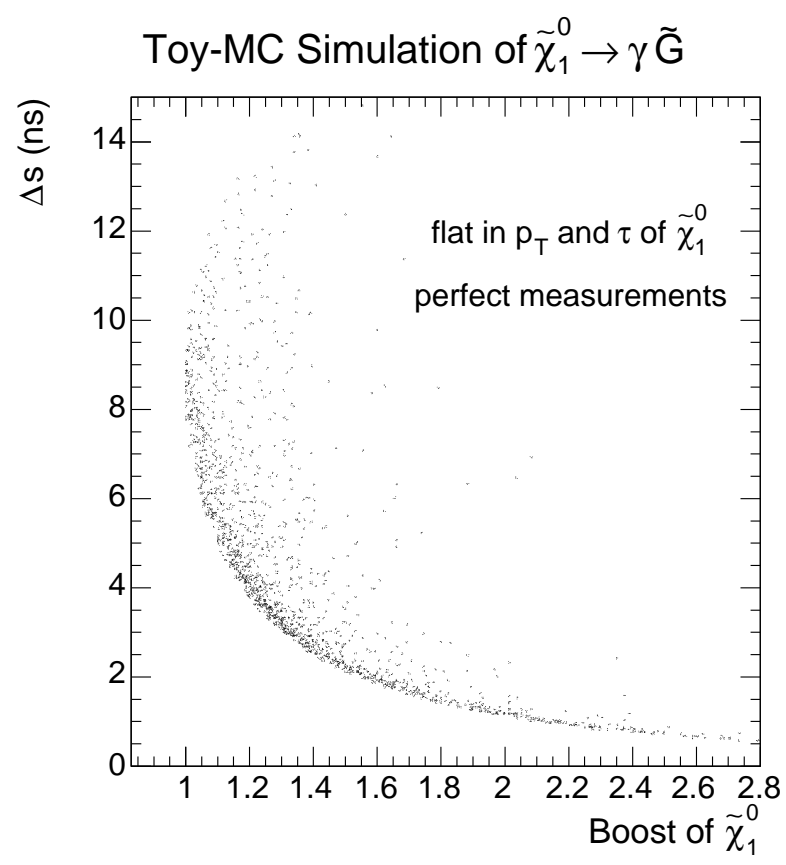

Figure 3: The $\Delta s$ distribution as a function of the boost of the neutralino for a lifetime "slice" of $8.5 \mathrm{~ns} \leq \tau_{\text {evt }, \mathrm{L}} \leq 9.0 \mathrm{~ns}$. In the region $1.0<$ boost $<1.5$ neutralinos remain in the detector and can produce a large $\Delta s$. Neutralinos with high boost, that is high $p_{T}$, are more likely to leave the detector or, if they don't, produce low $\Delta s$. Thus, events with the largest $\Delta s$ are produced by neutralinos with large lifetimes and low boosts.

\section{NEUTRALINO PAIR PRODUCTION AS A MEASURE OF QUASI-MODEL-INDEPENDENT SENSITIVITY}

\section{A. Analysis Methods and their Efficiency as a Function of Neutralino Mass and Lifetime}

Here we estimate our sensitivity to neutral, long-lived particles which decay to photons in as model-independent a manner as possible. We do so by considering a GMSB model [15] which we restrict to direct neutralino pair production and decay: $p \bar{p} \rightarrow \tilde{\chi}_{1}^{0} \tilde{\chi}_{1}^{0} \rightarrow \gamma \tilde{G} \gamma \tilde{G}$. We use the PYTHIA [16] event generator, with ISAJET [17] to generate the SUSY masses, and PGS with the parameter file for the CDF detector 18] as a simple detector simulation, modified for the use of timing information. We accept photons with a rapidity $|\eta| \leq 2.1$ and a transverse energy $E_{T} \geq 12 \mathrm{GeV}$ according to the CDF/EMTiming fiducial region and trigger [1, 2]. We first look at the efficiency of the timing system with infinite resolution as a function of neutralino mass and lifetime for different $\Delta s$ restrictions. Then we discuss background estimations, take into account a timing resolution of $1.0 \mathrm{~ns}$ and find the sensitivities for the model predictions for both the single and diphoton analysis.

Figure 5 shows the efficiency versus neutralino lifetime for a mass of $70 \mathrm{GeV} / c^{2}[19]$ for events with $\Delta s \geq 0 \mathrm{~ns}$ (photons from neutralinos remaining in the detector) and events 


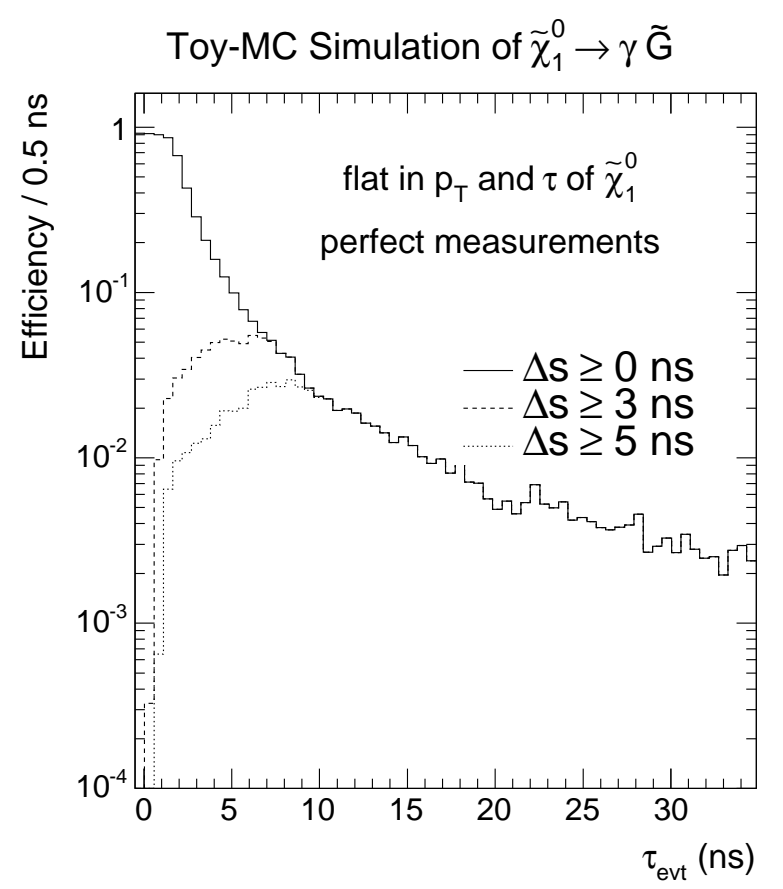

Figure 4: The efficiency as a function of the event lifetime, $\tau_{\text {evt }}$, of the neutralino. We distinguish between events in which the neutralino remains in the detector, and events with photons of medium and large $\Delta s$. The efficiency is $100 \%$ for prompt decays (a small difference shows up as a binning effect) for a photon to be identified, but only a very small efficiency for events with low $\tau_{\text {evt }}$ at large $\Delta s$. At large $\tau_{\text {evt }}$ only few events stay in the detector, however if a neutralino is long-lived and stays in the detector, it has large $\Delta s$. We note that the true efficiency shape depends on the production mechanism i.e. the neutralino $p_{T}$ distribution.

with a $\Delta s \geq 5 \mathrm{~ns}$, separated into single and diphoton events. We get essentially the same shapes as in Fig. 4 however the overall efficiency is less as we now consider the exponentially distributed neutralino lifetime instead of an event lifetime. For all four distributions there is an efficiency maximum in the lifetime region between 4 and 9 ns. At lower lifetimes the probability that the neutralino stays in the detector is large enough that the diphoton final state dominates. For any $\Delta s>0$ requirement the efficiency is zero at $\tau_{\tilde{\chi}}=0 \mathrm{~ns}$, since all photons have $\Delta s=0$. At a lifetime of about $3 \mathrm{~ns}$, independent of the $\Delta s$ cut, single photon events become dominant. At high lifetimes the efficiency decreases rapidly for both analyses as most of the neutralinos leave the detector. Hence, in order to have sensitivity in as much lifetime range as possible, we consider both $\gamma+\mathbb{H}_{T}$ and $\gamma \gamma+\mathbb{H}_{T}$ analyses.

In contrast the timing efficiency is essentially constant as a function of neutralino mass at a fixed lifetime. Figure [6] shows the efficiencies at $\tau_{\tilde{\chi}}=10 \mathrm{~ns}$, where the system has the highest efficiency and single photon events dominate. Note that the "dip" in the efficiency can be explained by the neutralino pair production mechanism: if the decay length is greater than the distance to the detector wall, the neutralino will leave. Since this is proportional to the ratio of the neutralino's transverse momentum to its mass, $\frac{p_{T}}{m}$, (at constant lifetime), the dip
Table I: The systematic uncertainties, estimated based on Refs. 3, 11], for luminosity, acceptance and number of background events for use in all analyses in estimating cross section limits.

\begin{tabular}{lc}
\hline \hline Factor & Syst. \\
\hline Luminosity & $5 \%$ \\
Acceptance & $10 \%$ \\
Number of background events & $30 \%$ \\
\hline \hline
\end{tabular}

occurs from a change in the shape of the $\frac{p_{T}}{m}$ distribution of the neutralinos as shown in Fig. [7 For a mass of $80 \mathrm{GeV} / c^{2}$ the maximum moves towards higher $\frac{p_{T}}{m}$ and the distribution broadens compared to $40 \mathrm{GeV} / c^{2}$, yielding a greater fraction of high- $p_{T}$ neutralinos and hence a loss in efficiency. As the mass gets higher the maximum remains the same and the distribution narrows, which in turn leads to a gain in efficiency. Thus, the efficiency is essentially independent of the neutralino mass, with slight variations originating from the production mechanism, specifically the neutralino momentum distribution.

\section{B. Backgrounds and Sensitivity to Neutralino Pair Production}

We now estimate the sensitivity of our system in a quasimodel-independent manner using the neutralino pair production introduced in the previous section, but taking into account SM backgrounds, more realistic cuts and the timing resolution. We consider separately single photon and diphoton events and present our sensitivity as the expected 95\% C.L. cross section upper limits for either case assuming no signal in the data. We also compare our results with the results of no EMTiming system available to estimate the effect of the EMTiming system over a set of kinematics-only selection requirements. Throughout this section we use the relative systematic uncertainties for luminosity, acceptance and background rates given in Table \and a $\Delta s$ resolution of $1.0 \mathrm{~ns}$. The expected cross section limits are calculated following 20] with the number of events observed "in the data" fluctuating around an expected mean background rate according to Poisson statistics. The cross section limit is, for a certain luminosity, a function of background events and signal acceptance, where both in turn are functions of specified cuts (e.g. $\Delta s$ and $E_{T}$ cuts in the $\gamma \gamma+\not_{T}$ case). By varying the cuts we find a signal acceptance and number of background events that, after smearing by systematic errors, minimizes the cross section limit.

$$
\text { 1. } \gamma \gamma+\not \phi_{T}
$$

A $\gamma \gamma+E_{T}$ analysis is expected to have the best sensitivity for low neutralino lifetimes. We follow the analysis in [3] (summarized in Table【I) and allow events in which both photons have $E_{T}>12 \mathrm{GeV}$ and $|\eta|<1$, and study final selection requirements on $\not_{T}$ and $\Delta s$. The background for this analysis consists of QCD events with fake $\not_{T}[21]$. We model the 
(a) GMSB $\tilde{\chi}_{1}^{0}$ pair production

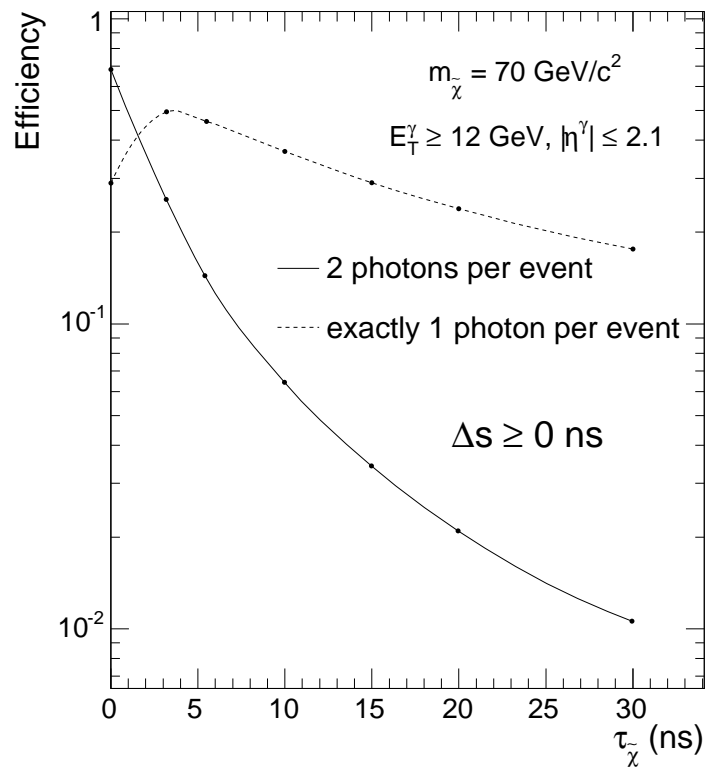

(b) GMSB $\tilde{\chi}_{1}^{0}$ pair production

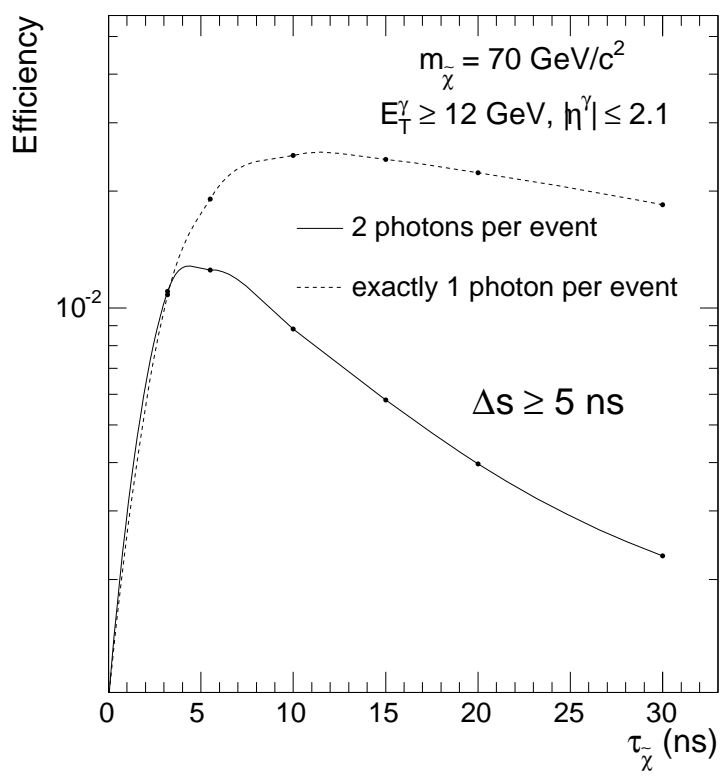

Figure 5: The efficiency for events to pass the various $\Delta s$ cuts (assuming perfect measurements) as a function of the neutralino lifetime at constant mass $\left(m_{\tilde{\chi}}=70 \mathrm{GeV} / c^{2}\right)$, separated into single and diphoton events at $\Delta s \geq 0$ ns and 5 ns. For any $\Delta s>0$ the efficiency is zero at $\tau_{\tilde{\chi}}=0 \mathrm{~ns}$, since all photons would have $\Delta s=0$. For high $\tau$ neutralinos have a higher probability to leave the detector. For any $\Delta s$ one can find an efficiency maximum at about 5-10 ns. Single photon events are preferred towards higher $\Delta s$ requirements and/or higher lifetimes, due to increasing probability for a photon to leave the detector. Thus, we expect a $\gamma \gamma+\not_{T}$ to provide the best sensitivity for very low lifetimes, and a $\gamma+\not_{T}$ analysis to be best for higher lifetimes. (a) GMSB $\tilde{\chi}_{1}^{0}$ pair production

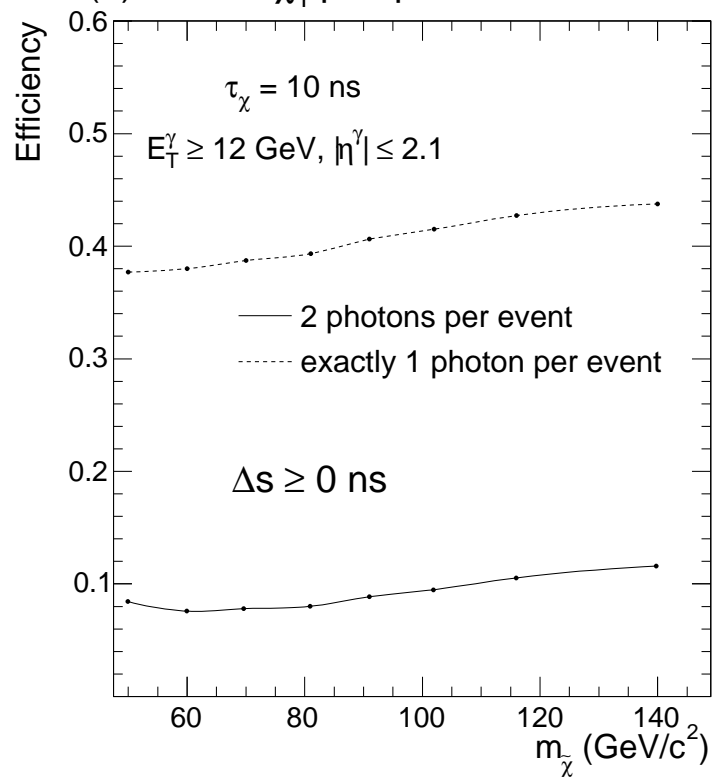

(b) GMSB $\tilde{\chi}_{1}^{0}$ pair production

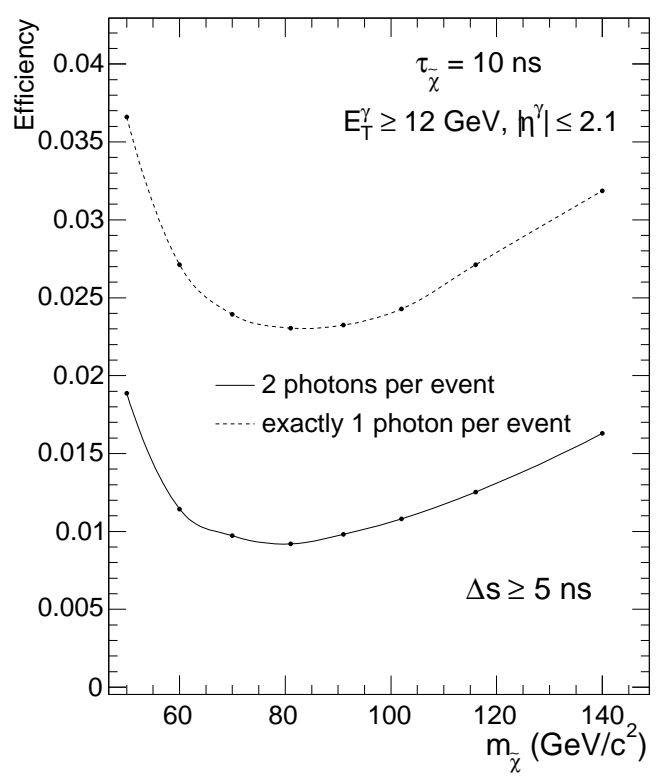

Figure 6: The efficiency as a function of the neutralino mass at a lifetime $\tau_{\tilde{\chi}}=10 \mathrm{~ns}$ for single and diphoton events at $\Delta s$ $\geq 0 \mathrm{~ns}$ and $5 \mathrm{~ns}$ (assuming perfect measurements). The ratio of single to diphoton events is independent of the neutralino mass and is roughly constant as a function of $\Delta s$. One can see a soft "dip" in the efficiency curve in a mass range of $40 \mathrm{GeV} / c^{2}-80 \mathrm{GeV} / c^{2}$. This effect is production dependent and due to a change in the $p_{T}$ distribution of the neutralinos (see Fig. [7). 


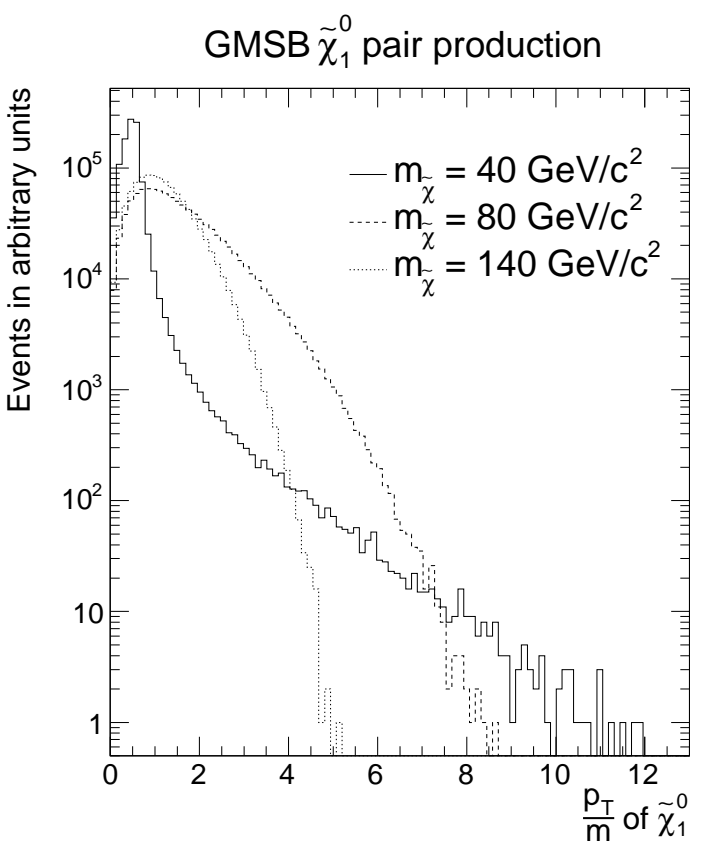

Figure 7: The neutralino $\frac{p_{T}}{m}$ distribution for masses $40 \mathrm{GeV} / c^{2}, 80 \mathrm{GeV} / c^{2}$ and $140 \mathrm{GeV} / c^{2}$ for neutralino pair production. For a mass of $80 \mathrm{GeV} / c^{2}$ the maximum moves towards higher $\frac{p_{T}}{m}$ and the distribution broadens compared to $40 \mathrm{GeV} / c^{2}$, yielding a greater fraction of high $p_{T}$ neutralinos which either leave the detector or produce low $\Delta s$ photons, and thus a loss in efficiency. For higher masses the maximum remains constant and the distribution narrows so the efficiency rises.

F $_{T}$ from QCD with a resolution of $10 \mathrm{GeV}$, i.e. we assume a measurement uncertainty of the transverse energy of all particles of $10 \mathrm{GeV}$ in each $x$ - and $y$-direction, as this reproduces well the numbers in [3] and allows us to extend our search region to large values of $\boldsymbol{H}_{T}$. Since all photons from QCD are promptly produced, we model them with a mean $\Delta s$ of $0 \mathrm{~ns}$, and a resolution of $1.0 \mathrm{~ns}$. We found that adding the $\Delta s$ values, $\Delta s_{12}=\Delta s_{1}+\Delta s_{2}$, and selecting signal events with either large $E_{T}$ or large $\Delta s_{12}$, either of which is not SM-like, maximizes the separation of signal and background as shown in Fig. 8 The position of the cuts are optimized for each mass and lifetime to minimize the $95 \%$ C.L. cross section limit, and we find that both the $\Delta s_{12}$ and $\xi_{T}$ cuts are stable at around $7 \mathrm{~ns}$ and $50 \mathrm{GeV}$ for non-zero lifetimes. Without timing information we found the optimal $\not_{T}$ cut to also be around $50 \mathrm{GeV}$.

$$
\text { 2. } \gamma+\not_{T}+0 \text { jets }
$$

From efficiency considerations and since the signal does not produce jets at the parton level we expect a $\gamma+E_{T}+0$ jets analysis to yield the best sensitivity for longer neutralino lifetimes. We follow the analysis in [10] (summarized in Table III and require the highest $E_{T}$ photon to have $E_{T}>25 \mathrm{GeV}$ and $|\eta| \leq 1$, a minimum $E_{T}$ of $25 \mathrm{GeV}$, and no jets or additional photons with $E_{T}>15 \mathrm{GeV}$, and study
Table II: The background and baseline selection criteria used for the $\gamma \gamma+\not \phi_{T}$ analysis following Ref. 3, 21].

\begin{tabular}{l}
\hline \hline Baseline selection requirements: \\
\hline$E_{T}^{\gamma_{1}}>12 \mathrm{GeV}, E_{T}^{\gamma_{2}}>12 \mathrm{GeV}$ \\
$\left|\eta^{\gamma_{1}}\right|<1,\left|\eta^{\gamma_{2}}\right|<1$ \\
\hline \hline Backgrounds: \\
\hline 2,577 events $/ 100 \mathrm{pb}^{-1}$ from QCD \\
$\Delta s_{12}=\Delta s^{\gamma_{1}}+\Delta s^{\gamma_{2}}, \overline{\Delta s_{12}}=0 \mathrm{~ns}$ with resolution $\sigma_{\Delta s_{12}}=$ \\
$1.41 \mathrm{~ns}$ \\
$E_{T}:$ Rayleigh distribution (Square-root of the sum of 2 Gaus- \\
sians squared) with $\sigma=10 \mathrm{GeV}$ \\
\hline \hline Optimization: \\
\hline Accept events where the event has a $\not_{T}$ greater than the \\
optimized $\mathscr{E}_{T}$ cut or whose photon has a $\Delta s_{12}$ greater than \\
the optimized $\Delta s_{12}$ cut. \\
\hline \hline
\end{tabular}

the final selection requirements on $E_{T}$ and $\Delta s$. The background for this analysis is dominantly QCD, $Z \gamma$ and cosmic ray sources. Since photons from cosmic ray sources hit the detector with no correlation between the arrival time and the time of collision, we expect them to be randomly distributed over time and model this with a flat random distribution in $\Delta s$. As in the previous section the $\Delta s$ of all other SM sources is dominated by the timing resolution of $1.0 \mathrm{~ns}$. The $\mathbb{E}_{T}$ for the backgrounds are modeled according to the shapes in [10], and extrapolated to large values using an exponential fit. The expected background and signal shapes are shown in Fig. 9] The final cuts on $\not_{T}$ and $\Delta s$ are applied to sort out events with a large $\not_{T}$ and whose photon has a $\Delta s$ within a lower bound and an upper bound: $\Delta s_{1} \leq \Delta s \leq \Delta s_{2}$, to reject photons from SM background as well as from cosmic ray sources. We find the optimized cuts at around $\Delta s_{1}=-2.0 \mathrm{~ns}, \Delta s_{2}=2.0 \mathrm{~ns}$ and $\not_{T}=80 \mathrm{GeV}$. However, $\Delta s_{2}$ could vary up to $3 \mathrm{~ns}$ for high lifetimes, $E_{T}$ up to $120 \mathrm{GeV}$ for higher masses. Without timing information we found the optimal $\mathbb{E}_{T}$ cut mostly at around $100 \mathrm{GeV}$.

\section{Results}

Figures [10] and 11] show the expected $95 \%$ C.L. cross section upper limits vs. $\tau_{\tilde{\chi}}$ for $m_{\tilde{\chi}}=70 \mathrm{GeV} / c^{2}$ and vs. $m_{\tilde{\chi}}$ for $\tau_{\tilde{\chi}}=20 \mathrm{~ns}$ for both analyses for a luminosity of $2 \mathrm{fb}^{-1}$. Figure 12 shows the ratio of the lower of the two $95 \%$ C.L. cross section limits with EMTiming system usage and without in two dimensions. In these plots we see four trends: 1) As a function of lifetime the cross section limits rise since the probability that the neutralinos decay in the detector goes down, 2) at high lifetimes the timing handle is better able to separate the signal from the backgrounds and produces better limits relative to kinematics alone, 3) as a function of mass the cross section decreases as more and more events pass the kinematic threshold, and 4) at low masses the timing handle is better able to separate the signal from the backgrounds because the momentum distribution happens be lower on average (see Fig. 8; which shows an example where the kinematics are such that there is only small additional acceptance from 
(a) Signal for $\gamma+\mathbb{E}_{\mathrm{T}}$ at $\mathrm{m}_{\overline{\mathrm{z}}}=70 \mathrm{GeV} / \mathrm{c}^{2}$

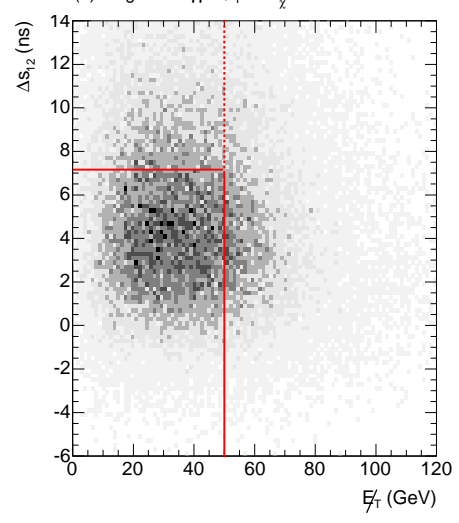

(b) Background for $\gamma \gamma+\mathbb{E}_{\mathrm{T}}$

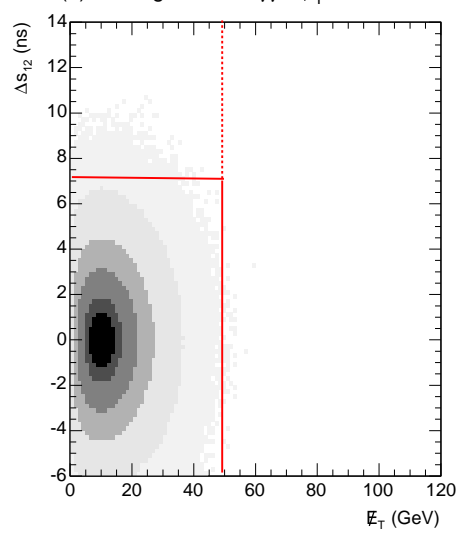

(c) Signal for $\gamma \gamma+Z_{T}$ at $m_{\gamma}=130 \mathrm{GeV} / \mathrm{c}^{2}$

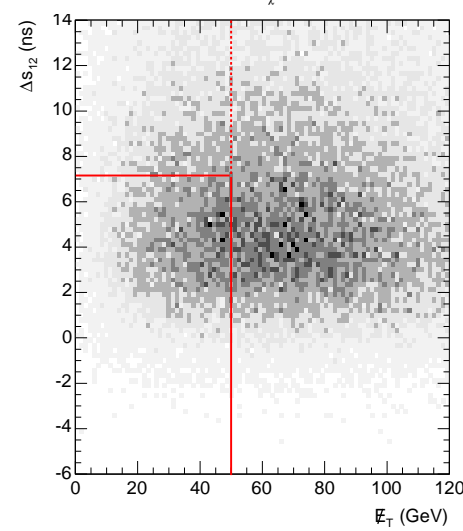

Figure 8: The distribution of $\Delta s_{12}$ and $\not_{T}$ for signal and background in the $\gamma \gamma+\not_{T}$ analysis. The distributions are (a) from direct neutralino pair production, with $m_{\tilde{\chi}}=70 \mathrm{GeV} / c^{2}$ and $\tau_{\tilde{\chi}}=10 \mathrm{~ns}$, (b) QCD background and (c) direct neutralino pair production, with $m_{\tilde{\chi}}=130 \mathrm{GeV} / \mathrm{c}^{2}$ and $\tau_{\tilde{\chi}}=10 \mathrm{~ns}$. The solid and dashed lines show the cuts with and without EMTiming system usage respectively that give the smallest $95 \%$ C.L. cross section limit. In (c) the mass is so large that there is only small additional acceptance from allowing large $\Delta s_{12}$ events which is why the sensitivity is not improved in this mass region (see 10 ).

Table III: The background and baseline selection criteria for the $\gamma+\mathbb{E}_{T}+0$ jets analysis following Ref. [10].

\begin{tabular}{l}
\hline \hline Baseline selection requirements: \\
\hline$E_{T}^{\gamma}>25 \mathrm{GeV}$ \\
$\left|\eta^{\gamma}\right|<1.0$ \\
$E_{T}>25 \mathrm{GeV}$ \\
No jets or additional photons with $E_{T}^{j e t}>15 \mathrm{GeV}$ \\
\hline \hline Backgrounds: \\
\hline 12.6 events / $100 \mathrm{pb}^{-1}$ from $Z \gamma \rightarrow \nu \bar{\nu} \gamma, \mathrm{W} \gamma, W \rightarrow e \nu, \mathrm{QCD}$ \\
and cosmics \\
Non-cosmics: $\Delta s=0, \overline{\Delta s_{12}}=0 \mathrm{~ns}$ with resolution $\sigma_{\Delta s}=$ \\
1.0 ns \\
Cosmics: $57.2 \%$ of total background, flat distribution in $\Delta s$ \\
$\not_{T}$ distribution according to [10], and extrapolated using an \\
exponential \\
\hline \hline Optimization: \\
\hline Accept events where the event has a $\not_{T}$ greater than the \\
optimized cut $\not_{T}$ and whose photon has a $\Delta s$ within a range \\
of optimized cuts $\Delta s_{1} \leq \Delta s \leq \Delta s_{2}$. \\
\hline \hline
\end{tabular}

allowing large $\Delta s_{12}$ events). As expected the $\gamma \gamma+\not{ }_{T}$ analysis yields lower cross sections when the mass or the lifetime is low (see Fig. 12). The ratio is greatest in this region and occurs at a mass around $50 \mathrm{GeV} / c^{2}$ and a lifetime of $10-20 \mathrm{~ns}$. The $\gamma+\not_{T}+0$ jets analysis yields lower cross section limits for the rest of the considered lifetime range and masses, but it is important to note that the course of the separation line of the analyses depends on the production momentum distribution of the neutralino. Unfortunately this analysis cannot be applied to searches for long-lived NLSP neutralinos in a true GMSB model with the preferred production processes as there the neutralinos are produced as part of cascades from gaugino pairs which contain jets. Therefore, we do a separate analysis for a full GMSB production in the next section.

\section{SENSITIVITY TO GMSB MODELS}

We next consider the sensitivity to full GMSB production where we allow all processes to contribute to the final state according to their predicted cross sections. We use the same simulation tools as in Section IIIA with the GMSB parameters chosen according to the Snowmass Slope guidelines 15] in the range where the neutralino is the NLSP. Again we consider a single photon and a diphoton analysis. The $\gamma \gamma+\not_{T}$ analysis methodology is identical to the case of neutralino pair production, but the single photon analysis must be modified to allow jets as here the neutralinos are part of cascades from gauginos which produce additional particles which, in general, could be identified jets. We thus use a $\gamma+\not_{T}+$ jets analysis.

$$
\text { A. } \gamma+\phi_{T}+\text { jets }
$$

A $\gamma+\not_{T}+$ jets analysis should be most sensitive to neutralinos with long lifetime which are produced in association with other particles in the final state such as from gaugino pair production. We follow the analysis in 11] (summarized in Table IV] and require events with the primary (highest $E_{T}$ ) photon to have $E_{T}>25 \mathrm{GeV}$ and $|\eta|=[0,1.1]$ or [1.5, 2.0], $E_{T}>25 \mathrm{GeV}, \geq 2$ jets of $E_{T}>20 \mathrm{GeV}$ and $|\eta|<2.0$, and study the final selection requirements on $E_{T}$ and $\Delta s$. The backgrounds are dominated by QCD and $\mathrm{W}+$ jets [22]. The expected $\mathscr{E}_{T}$ of the background is modeled according to Ref. 11], and since the backgrounds are from SM we take a mean $\Delta s=0 \mathrm{~ns}$ with a resolution of $1.0 \mathrm{~ns}$. The signal and background shapes are shown in Fig. 13] We find that the optimal final selection requirements accept events in which the event has a large $\mathbb{F}_{T}$ or a large $\Delta s$. Again, both cuts are optimized to minimize the $95 \%$ C.L. cross section limit for each mass and lifetime case. For without-timing usage we find the 
(a) Signal for $\gamma+\mathbb{E}_{T}+0$ jets

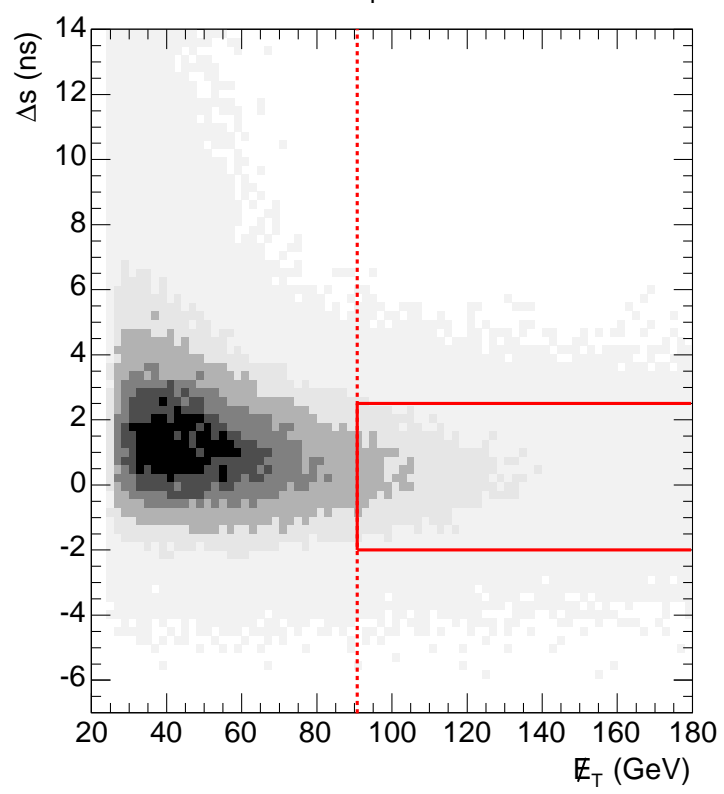

(b) Background for $\gamma+\mathbb{E}_{T}+0$ jets

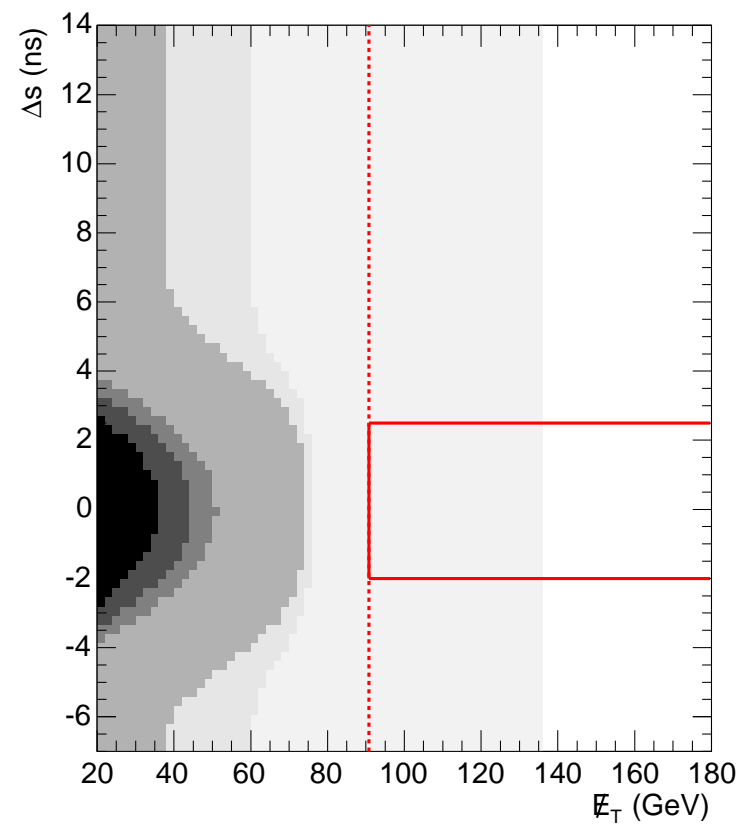

Figure 9: The distribution of $\Delta s$ and $\not_{T}$ for signal and background in the $\gamma+\mathbb{E}_{T}+0$ jets analysis. The distributions are (a) from direct neutralino pair production, with $m_{\tilde{\chi}}=70 \mathrm{GeV} / c^{2}$ and $\tau_{\tilde{\chi}}=10 \mathrm{~ns}$, and (b) from SM background. The solid and dashed lines show the cuts with and without EMTiming system usage respectively that give the smallest $95 \%$ C.L. cross section limit.
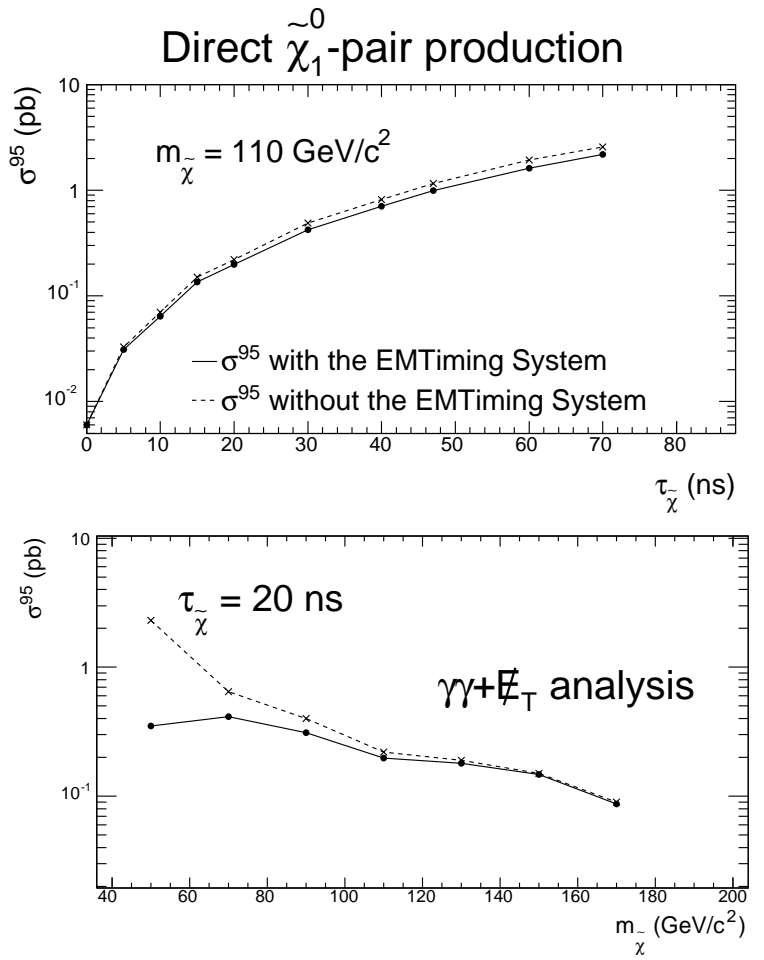

Figure 10: The expected 95\% C.L. cross sections limits on neutralino pair production as a function of $\tau_{\tilde{\chi}}$ for $m_{\tilde{\chi}}=70 \mathrm{GeV} / c^{2}$ (top) and as a function of $m_{\tilde{\chi}}$ for $\tau_{\tilde{\chi}}=20 \mathrm{~ns}$ (bottom) in the $\gamma \gamma+\mathbb{E}_{T}$ analysis for $2 \mathrm{fb}^{-1}$ luminosity both with and without a timing system for comparison. As expected at $\tau_{\tilde{\chi}}=0 \mathrm{~ns}$ the cross sections merge as the timing system has no effect; for higher $\tau_{\tilde{\chi}}$ the sensitivity goes down as more photons leave the detector, but the difference of the limits increases as $\Delta s$ gets larger for the signal and timing becomes more helpful. The limits get better as the mass goes up since more of the events pass the kinematic requirements, however the timing system only provides real additional sensitivity at the lowest masses where the neutralino momentum distribution is softer.

$E_{T}$ cut to be around $50 \mathrm{GeV}$ for masses around $70 \mathrm{GeV} / c^{2}$, varying up to $110 \mathrm{GeV}$ for masses around $150 \mathrm{GeV} / c^{2}$. For with-timing usage we find only a $\Delta s$ cut around $4 \mathrm{~ns}$ which is stable for all masses and lifetimes, and no $\not_{T}$ cut other than the baseline $\not_{T}>25 \mathrm{GeV}$ (except for $\tau_{\tilde{\chi}}=0 \mathrm{~ns}$ where the diphoton case has the best sensitivity). While it is outside of our ability to predict, one might find further optimization is possible by further lowering the baseline selection requirements.

\section{B. Results}

Figures 14 and [15] show the expected $95 \%$ C.L. cross section upper limits vs. $\tau_{\tilde{\chi}}$ for $m_{\tilde{\chi}}=70 \mathrm{GeV} / c^{2}$ and vs. $m_{\tilde{\chi}}$ for $\tau_{\tilde{\chi}}=20 \mathrm{~ns}$ for both analyses for a luminosity of $2 \mathrm{fb}^{-1}$. Figure [16] shows the ratio of the lowest 95\% C.L. cross section limits with EMTiming system usage and without in two 

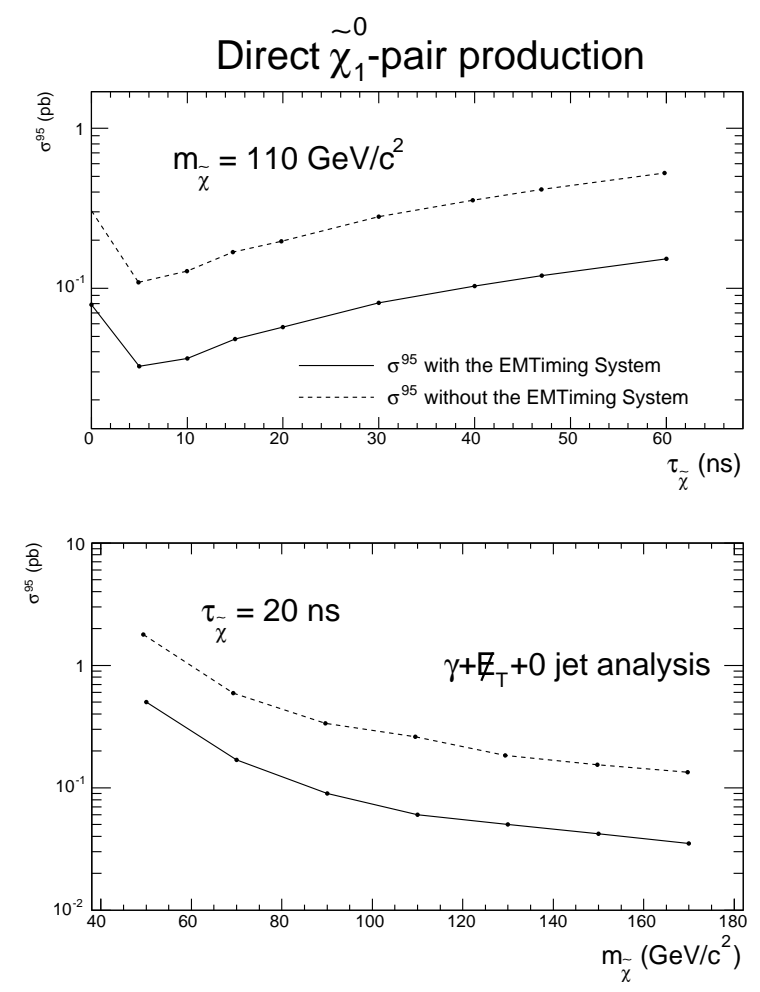

Figure 11: The expected 95\% C.L. cross section limits on neutralino pair production as a function of $\tau_{\tilde{\chi}}$ for $m_{\tilde{\chi}}=70 \mathrm{GeV} / c^{2}$ (top) and as a function of $m_{\tilde{\chi}}$ for $\tau_{\tilde{\chi}}=20 \mathrm{~ns}$ (bottom) in the $\gamma+E_{T}+0$ jets analysis for $2 \mathrm{fb}^{-1}$ both with and without a timing system. As in Fig. 10 for higher $\tau_{\tilde{\chi}}$ the sensitivity goes down as more photons leave the detector, but the difference of the limits increases as $\Delta s$ gets larger for the signal and timing becomes more helpful. The curves do not merge at 0 ns lifetime since cosmic ray backgrounds always contribute at high $\Delta s$ and the timing system always has some effect on the cross section limit. The rise from $10 \mathrm{~ns}$ to $0 \mathrm{~ns}$ originates in an increasing probability towards zero lifetime for two photons to remain in the detector, yielding lower efficiency for a single photon analysis. The limits get better as the mass goes up since more of the events pass the kinematic requirements.

dimensions. We see the same general trends as in neutralino pair production as the signal shapes are similar in both analyses.

A comparison of the cross section limits with the production cross sections in the GMSB model at a luminosity of $2 \mathrm{fb}^{-1}$ gives the mass vs. lifetime exclusion regions shown in Fig. 17 As expected, timing has the biggest effect at low masses and high lifetimes. We have also indicated the exclusion regions from LEP II from both direct and indirect searches [13], and the line $m_{\tilde{G}}=1 \mathrm{keV} / c^{2}$, below which is the theoretically preferred region from cosmological constraints 14]. LEP effectively excludes all neutralino masses under $80 \mathrm{GeV} / c^{2}$ up to high lifetimes, with a small extension to $100 \mathrm{GeV} / \mathrm{c}^{2}$ for lifetimes below $20 \mathrm{~ns}$. For $2 \mathrm{fb}^{-1}$, in run II, the Tevatron should significantly extend the sensitivity at large mass and lifetimes, covering most of the lifetimes for $m_{\tilde{G}}<1 \mathrm{keV} / c^{2}$ up to a mass of around $150 \mathrm{GeV} / c^{2}$. The

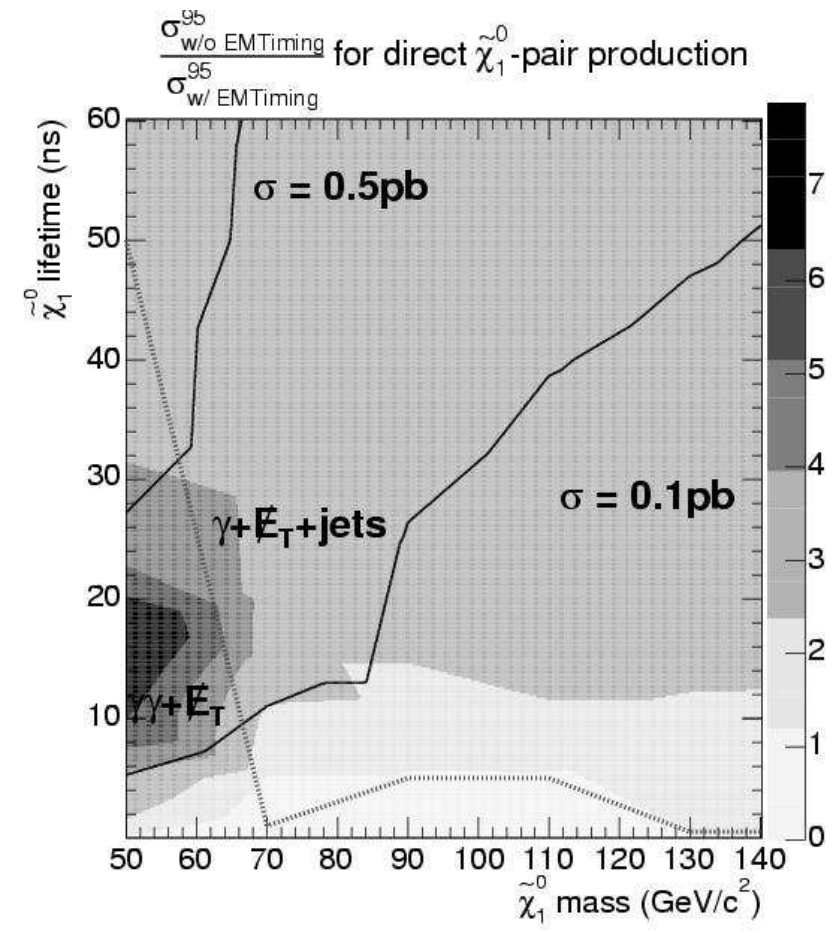

Figure 12: This plot combines the $\gamma \gamma+\not_{T}$ and $\gamma+E_{T}+0$ jets analysis results for neutralino pair production for $2 \mathrm{fb}^{-1}$ of data and is a 2-dimensional visualization of Figs. 10] and 11] The contours of constant cross section limit are shown as the solid lines, and the separation line between the regions where the two different analyses provide the best sensitivity is given by the dotted line; the $\gamma+\not_{T}+0$ jets analysis shows better cross section limits than a $\gamma \gamma+\mathbb{E}_{T}$ analysis in the mass and lifetime range above the dashed line. The shaded regions delineate the contours of the ratio of the 95\% C.L. cross section limits between with and without EMTiming information. The ratio is greatest for a low neutralino mass and a lifetime of 10-20 ns, and lowest for a high mass and low lifetime.

Table IV: The background and baseline selection criteria used for the $\gamma+\not_{T}+$ jets analysis following Refs. [11, 22].

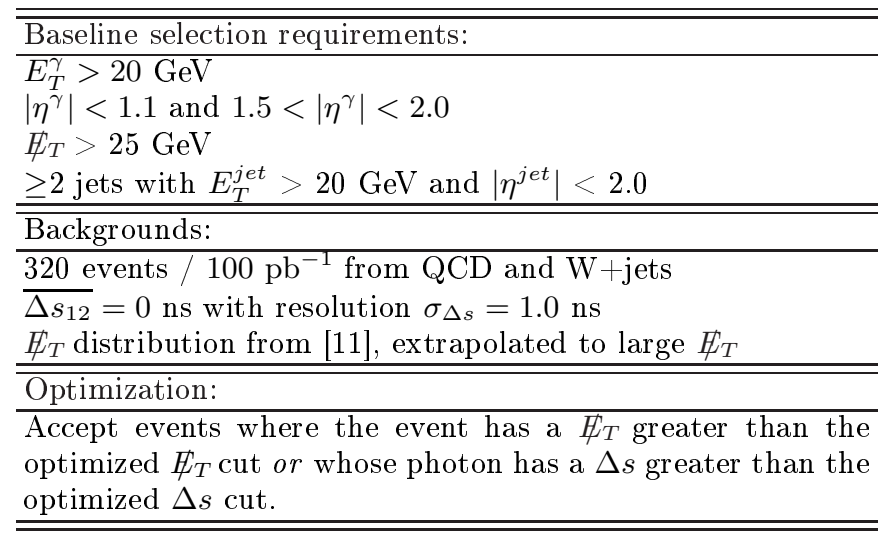


(a) Signal for $\gamma+\mathbb{E}_{T}+$ jets

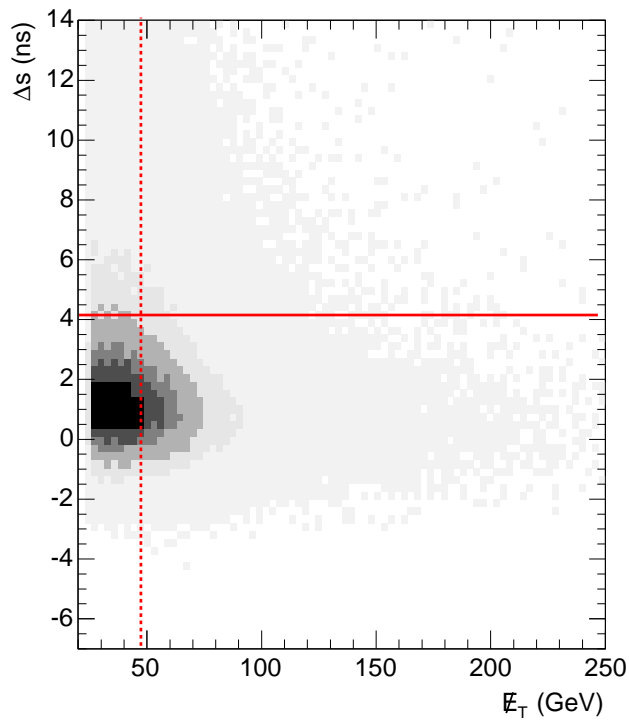

(b) Background for $\gamma+\mathbb{E}_{\mathrm{T}}+$ jets

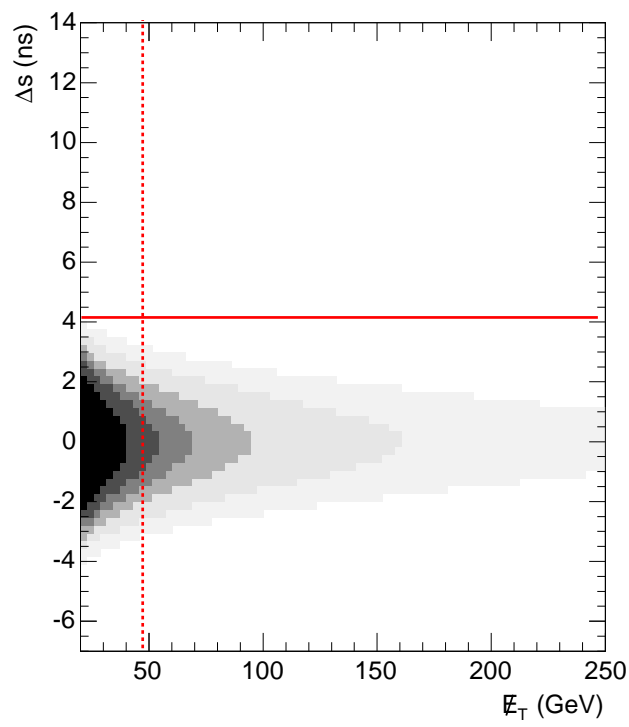

Figure 13: The distribution of $\Delta s$ and $E_{T}$ for signal and background in the $\gamma+\mathscr{E}_{T}+$ jets analysis. The distributions are (a) from full GMSB production, with $m_{\tilde{\chi}}=70 \mathrm{GeV} / c^{2}$ and $\tau_{\tilde{\chi}}=10 \mathrm{~ns}$, and (b) from SM background. The solid and dashed lines show the cuts with and without EMTiming system usage respectively that give the smallest $95 \%$ C.L. cross section limit.

mass exclusion limit at $168 \mathrm{GeV}$ for $\tau_{\tilde{\chi}}=0 \mathrm{~ns}$ is comparable to the limit presented in the $\mathrm{D} \varnothing$ study of displaced photons in Ref. 23], but for large lifetimes this result significantly extends the reach.
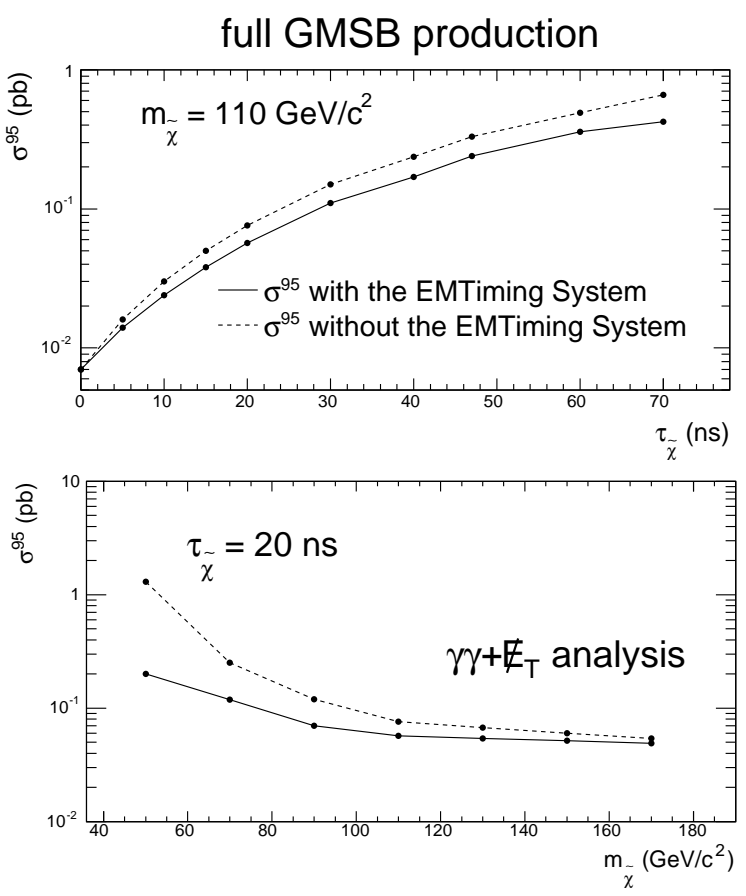

Figure 14: The expected 95\% C.L. cross section limits as a function of $\tau_{\tilde{\chi}}$ for $m_{\tilde{\chi}}=70 \mathrm{GeV} / c^{2}$ (top) and as a function of $m_{\tilde{\chi}}$ for $\tau_{\tilde{\chi}}=20 \mathrm{~ns}$ (bottom) at $2 \mathrm{fb}^{-1}$ for with and without EMTiming for full GMSB production in a $\gamma \gamma+\not_{T}$ analysis. The results are similar to those in Fig. 10]

\section{Factors that might change the cross section limit}

While we have taken the best available nominal values from the references for both the contamination of cosmic ray background events and the timing resolution in the $\gamma \gamma+\mathbb{E}_{T}$ and a $\gamma+E_{T}+$ jets analyses, the limits are sensitive to misestimations of these values. For simplicity, rather than include them as a systematic error we estimate the variation of our results for these effects on our cross section limits for a neutralino mass of $110 \mathrm{GeV} / c^{2}$ and a lifetime of $40 \mathrm{~ns}$ beyond the LEP II exclusion region. Figure 18 shows the cross section limit as a function of the fraction of events which are from cosmics in the background sample. Using the same analysis style as in the $\gamma+\mathbb{E}_{T}+0$ jets case we find cuts around $\Delta s_{1}=3.0 \mathrm{~ns}$ and $\not_{T}=55 \mathrm{GeV}$, with $\Delta s_{2}$ varying from infinity down to $7 \mathrm{~ns}$ with a rising fraction of cosmics. The limits rise approximately linearly as a function of the fraction of events which are from cosmics. An upper bound on the fraction of cosmics of $10 \%$ would reduce the limits by about a factor of four; a more reasonable estimate is probably $1-5 \%$ which would raise the limts by a factor of 2-3. The limits are potentially more sensitive to the resolution. Figure [19] shows how the limits change as a function of the system resolution for the same mass and lifetime, for the original $\gamma+E_{T}+$ jets analysis. While there is a mass/lifetime dependent resolution threshold from where the limit can change drastically and approach the cross section without EMTiming, the limits are fairly stable (with the same factor of 2) for resolutions within 


\section{full GMSB production}
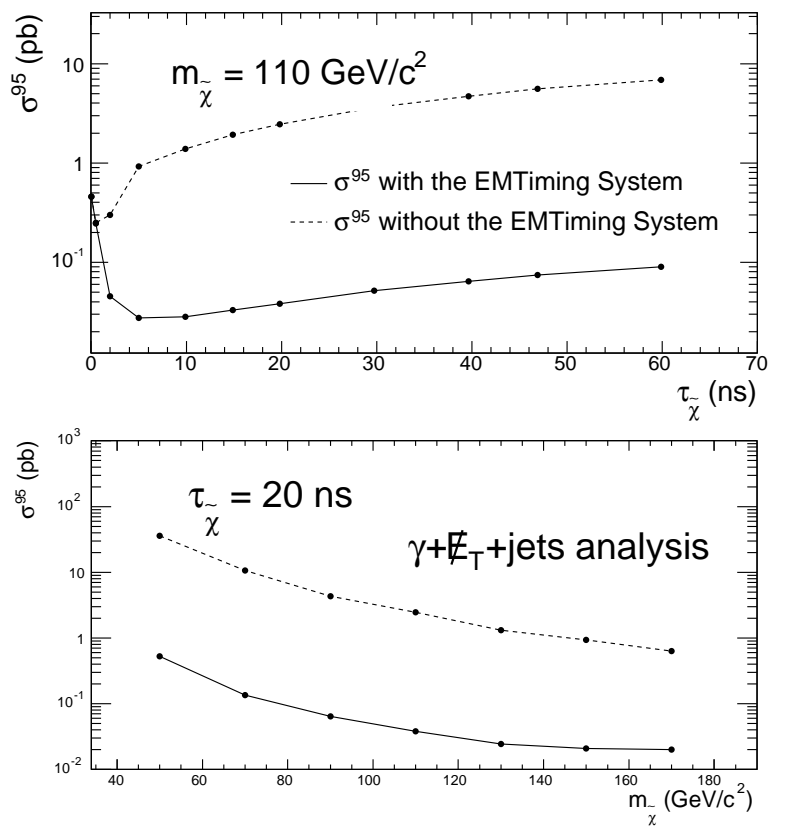

Figure 15: The expected 95\% C.L. cross section limits as a function of $\tau_{\tilde{\chi}}$ for $m_{\tilde{\chi}}=70 \mathrm{GeV} / c^{2}$ (top) and as a function of $m_{\tilde{\chi}}$ for $\tau_{\tilde{\chi}}=20 \mathrm{~ns}$ (bottom) at $2 \mathrm{fb}^{-1}$ for with and without EMTiming System for full GMSB production in a $\gamma+\not \phi_{T}+$ jets analysis. For all but the lowest lifetimes the timing information significantly improves the cross section limits. Note that here the cross sections merge at zero lifetime since we have neglected cosmics in this analysis following [11, 22].

$20 \%$ of the nominal $1 \mathrm{~ns}$ resolution, and there is good reason to believe that the resolution will be better than advertised 7].

\section{CONCLUSION}

We have studied the prospects of using the new EMTiming system at CDF in the search for neutral, long-lived particles which decay to photons, as one can find in supersymmetric models. We find that the kinematic requirements and the EMTiming system provide excellent rejection against SM backgrounds in complementary fashion. As the mass increases the kinematics are more important and the sensitivity gets better. For a given mass, as the lifetime increases more and more of the neutralinos leave the detector and the overall sensitivity goes down, but the EMTiming system provides additional rejection power and allows for significant exclusions even at large lifetimes. While the region where EMTiming produces the most additional rejection is already excluded by LEP II, the additional handle it provides should allow the Tevatron in run II to produce the world's most stringent limit at masses above $80 \mathrm{GeV} / c^{2}$ at high lifetimes and has the potential to cover the entire region for $m_{\tilde{G}}<1 \mathrm{keV} / c^{2}$ up to a neutralino mass of around $150 \mathrm{GeV} / c^{2}$.

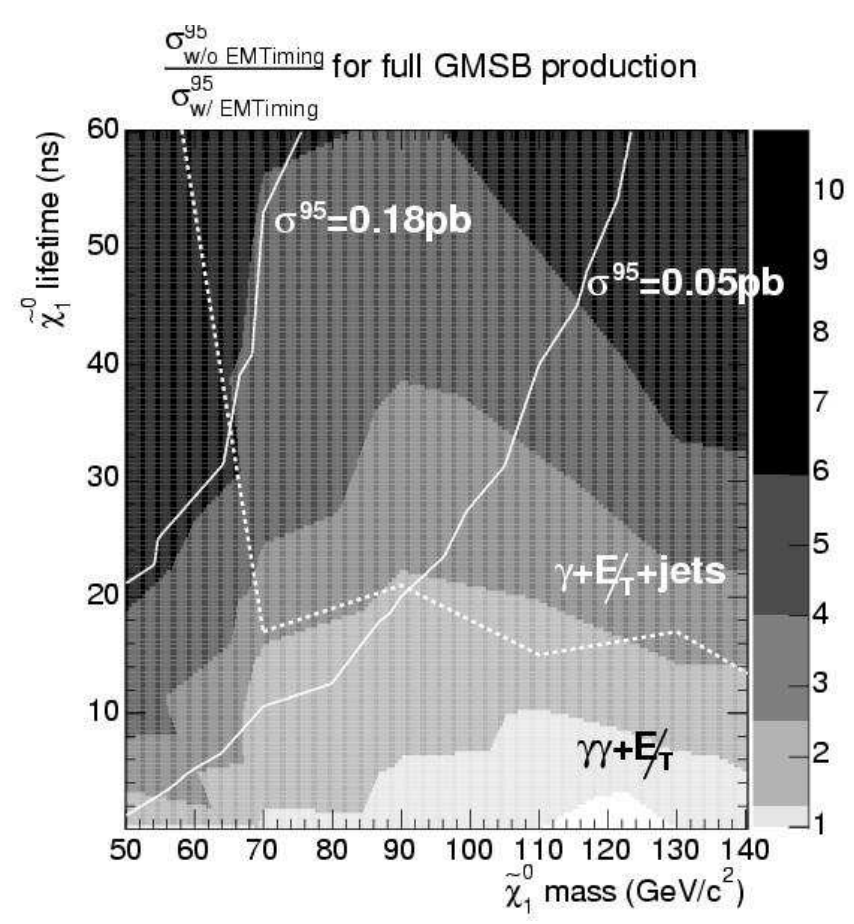

Figure 16: This plot combines the $\gamma \gamma+\not_{T}$ and $\gamma+\mathbb{E}_{T}+$ jets analysis results for a full GMSB model simulation for $2 \mathrm{fb}^{-1}$ of data and is a 2-dimensional visualization of Figs. [14 and 15] The contours of constant cross section limit are shown as the solid lines, and the separation line between the regions where the two different analyses provide the best sensitivity is given by the dotted line; the $\gamma+\not_{T}+$ jets analysis shows better cross section limits than a $\gamma \gamma+\mathbb{E}_{T}$ analysis in the mass and lifetime range above the dashed line. The shaded regions delineate the contours of constant ratio of the $95 \%$ C.L.- cross-section limits between with and without EMTiming information. The EMTiming system has its most effective region at high lifetime and is least effective for high masses, where the kinematics give the best separation.

\section{Acknowledgments}

The authors would especially like to thank T. Kamon for his early contributions to the genesis of the idea of using the EMTiming system at CDF to search for GMSB SUSY; it was his preliminary feasibility study which showed that the analysis might be possible. We would also like to thank R. Arnowitt, R. Culbertson, B. Dutta, A. Garcia-Bellido, M. Goncharov, V. Khotilovich, V. Krutelyov, S. W. Lee, P. McIntyre and S. Mrenna for help and useful discussions. We thank Fermilab and the FNAL CAF system administrators for providing additional computing power. This research was generously supported using funding from the College of Science and the Department of Physics at Texas A\&M University.

\section{Appendix A: PHOTON POINTING}

As shown in Fig. 17 LEP II has already excluded the low neutralino mass region using a photon pointing method [13]. 
(a)

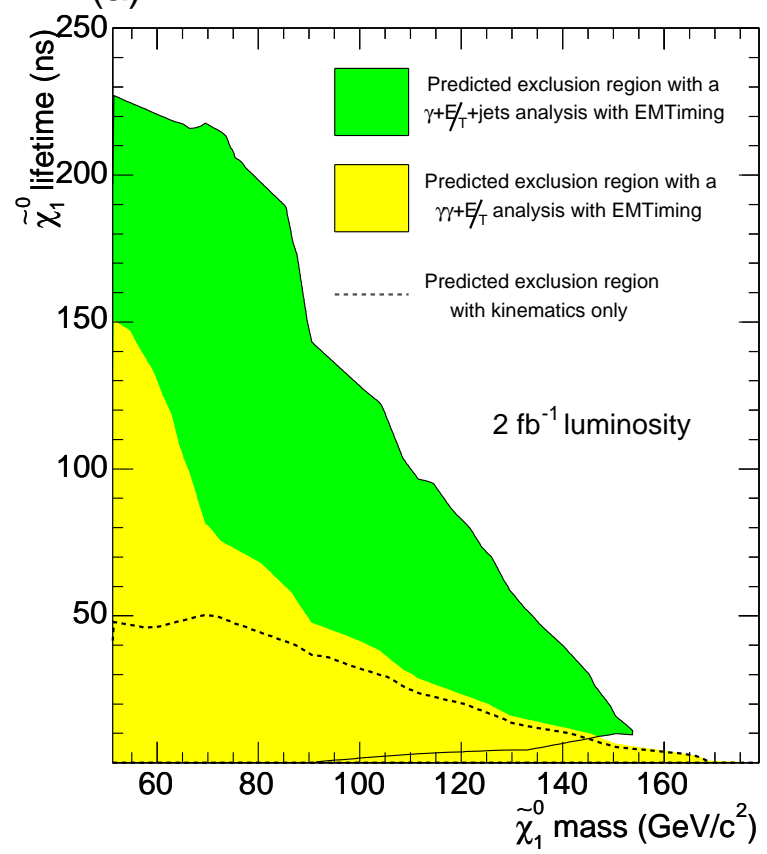

(b)

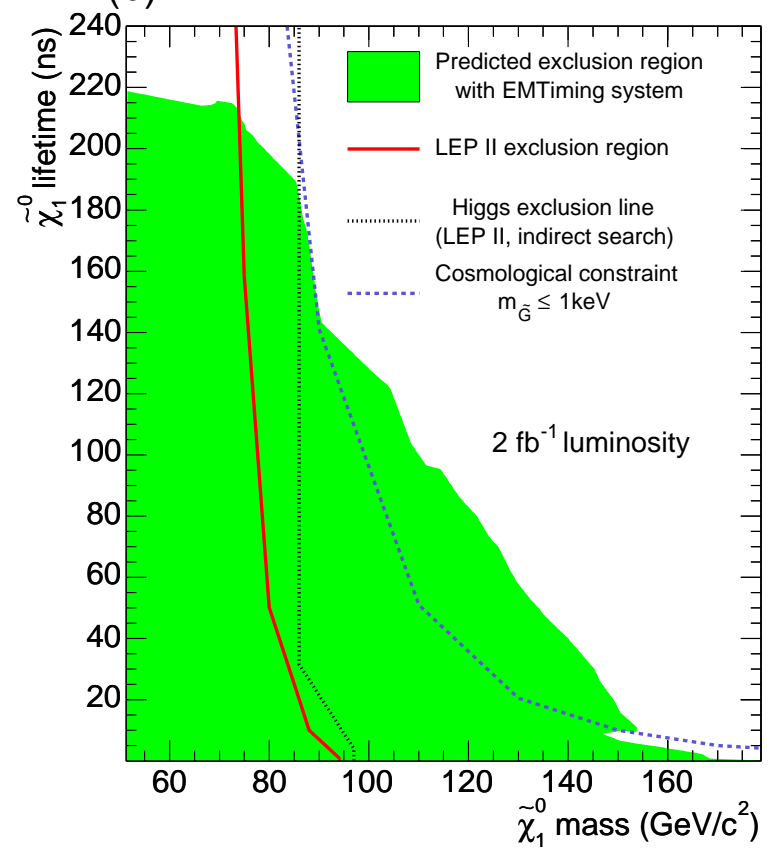

Figure 17: The expected 95\% C.L. exclusion regions as a function of neutralino lifetime and mass for full GMSB production at $2 \mathrm{fb}^{-1}$ luminosity for the the $\gamma \gamma+\not_{T}$ and a $\gamma+\not_{T}+$ jets analysis separately. In plot (a) the region below the dashed line is the exclusion region from kinematics alone, i.e., where no timing information is used. Plot (b) shows the full exclusion region from the overlap of the two analyses and compares the results to the direct and indirect search limits from LEP II [13] and the theoretically favored region from cosmological constraints $\left(m_{\tilde{G}}<1 \mathrm{keV} / \mathrm{c}^{2}\right)$ 14]. The Tevatron in run II should be able to significantly extend the LEP II limits and provide sensitivity in the favored region for all masses below about $150 \mathrm{GeV} / c^{2}$.

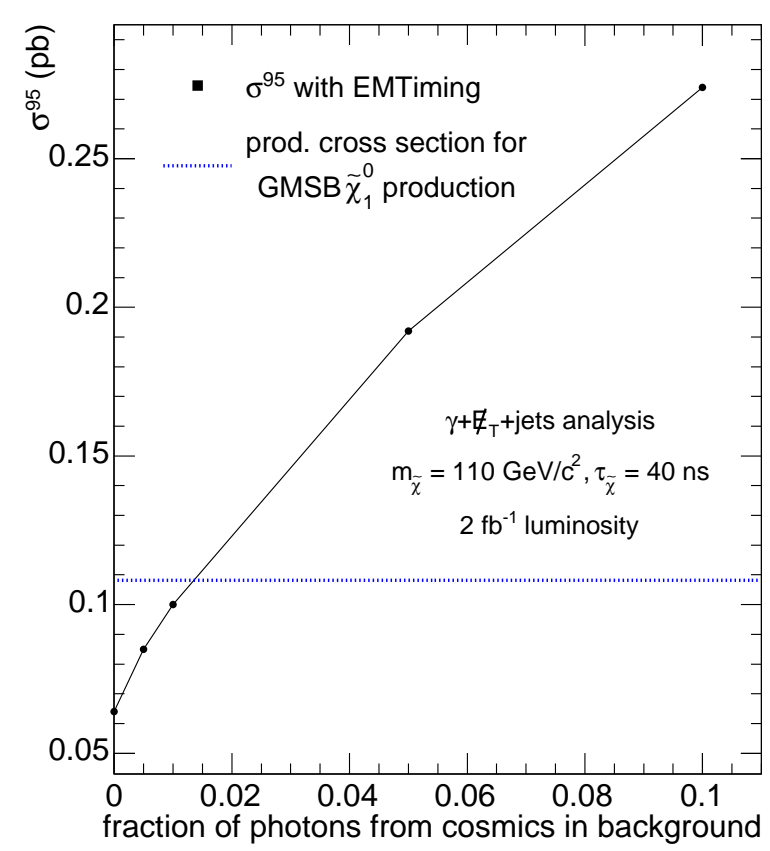

Figure 18: The $95 \%$ C.L. cross section limit on full GMSB production at $m_{\tilde{\chi}}=110 \mathrm{GeV} / c^{2}$ and $\tau_{\tilde{\chi}}=40 \mathrm{~ns}$ as a function of the fraction of the background from cosmic ray sources for a $\gamma+\not_{T}+$ jets analysis. The cross section limits rise approximately linearly as a function of the fraction and $10 \%$ provides an outer bound on this fraction. A more reasonable fraction is probably $1-5 \%$ which roughly doubles the limit.

In this section we compare the EMTiming system to a potential photon pointing ability at CDF. A non-zero lifetime can result in a macroscopic decay length and impact parameter, where the impact parameter of the photon is basically the closest distance of the trajectory to the collision point. While CDF has never used its calorimeter for a pointing measurement it is possible to use the central EM strip/wire gas chamber (CES) and the central pre-radiator gas chamber (CPR) at CDF to measure two points along the photon trajectories that determine the direction of the photon, and trace it back to yield the impact parameter [24]. Since the CPR has no $z$-measurement ability this allows only a measurement of the radial component of the impact parameter with an estimated resolution of $10 \mathrm{~cm}$ (see Table $\mathrm{V}$ ). One of the primary reasons this has not been used is the conversion, i.e. measurement, probability, of $\sim 65 \%$, with an angular dependence obtained with:

$$
P_{C}=1-e^{-\frac{N_{\mathrm{rad}}}{\sin \theta}}
$$

where $N_{\text {rad }}=1.072$ is the number of radiation lengths before the CPR and $\theta$ is the angle with respect to the beamline. To estimate the sensitivity with a pointing method we consider a $\gamma+\not_{T}+$ jets analysis. Figure 20 shows the distribution of the signal events vs. impact parameter and $\Delta s$ in a $\gamma+\#_{T}+$ jets analysis taking into account the measurement probability; there are roughly as many events in the region of low impact parameter and high $\Delta s$ as there are at high impact parameter and low $\Delta s$. Hence either method should have 


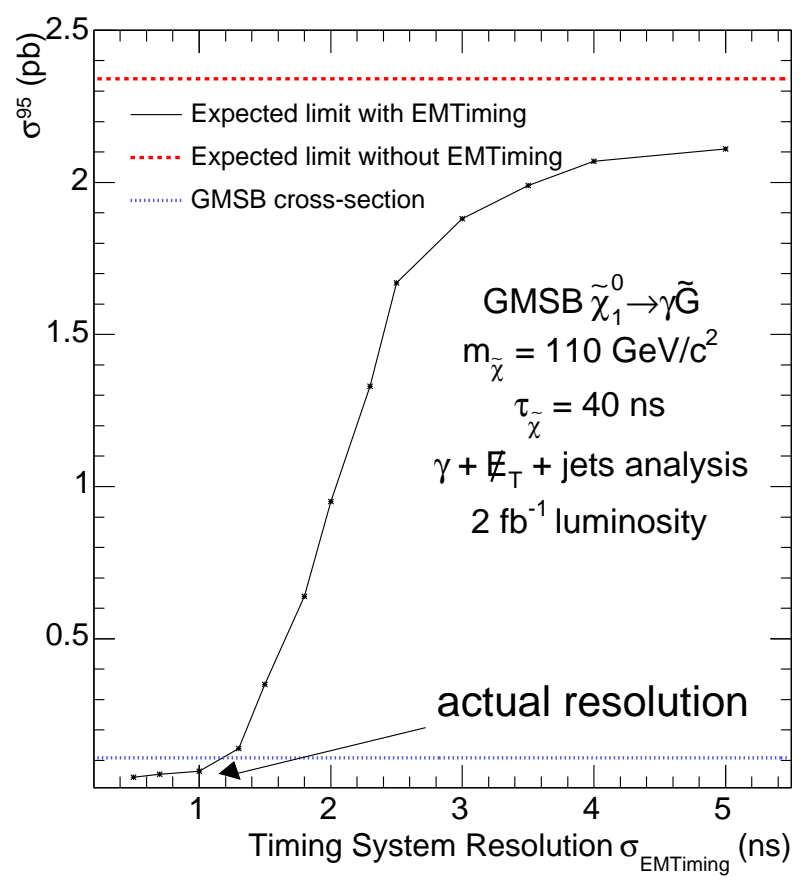

Figure 19: The $95 \%$ C.L. cross section limits vs. EMTiming system resolution for $m_{\tilde{\chi}}=110 \mathrm{GeV} / c^{2}$ and $\tau_{\tilde{\chi}}=40 \mathrm{~ns}$ at $2 \mathrm{fb}^{-1}$ luminosity in the $\gamma+\mathbb{E}_{T}+$ jets analysis. As expected, for large resolution the cross section with EMTiming approaches the cross section without EMTiming. A system resolution of $1.0 \mathrm{~ns}$ improves the cross section limit by a factor of about 20, but this varies as a function of mass, lifetime and depends on the analysis. It is reasonable to assume that the resolution will be within $20 \%$ of the nominal $1.0 \mathrm{~ns}$ presented here.

roughly the same effect on the exclusion region, as confirmed by Fig. 21 which shows the expected exclusion region in the mass-lifetime plane. While timing is better than pointing by itself, if pointing turns out to be feasible, a combination of the two would improve the sensitivity.

Considered separately, a second advantage of timing is that it "filters" manifestly long lifetime events, whereas the impact parameter allows also short lifetime-high momentum events, which might come from SM. Another possible advantage of the combination is that in the event of an excess, we could draw more information about the individual events, for instance determine the direction of the photon. With the $x$ $y$-direction being fixed by the CPR/CES measurement, we can use the timing system to measure the $z$-direction. Or if the pointing provided the photon direction in $z$ and $x$ - $y$, one could possibly determine the position of the vertex and thus the decay time. However, with the current $1.0 \mathrm{~ns}$ resolution the photon vertex position resolution would be roughly $50 \mathrm{~cm}$, if we assume the neutralino boost to be $\sim 1.0$.
Table V: Photon pointing parameters for the CDF detector [24]. With this combination we estimate an impact parameter resolution of $10 \mathrm{~cm}$ in the radial direction.

\begin{tabular}{lr}
\hline \hline measurement only in radial direction \\
Radius of CES & $184.15 \mathrm{~cm}$ \\
Radius of CPR & $168.29 \mathrm{~cm}$ \\
$\sigma_{\mathrm{CES}}$ & $2 \mathrm{~mm}$ \\
$\sigma_{\mathrm{CPR}}$ & $5 \mathrm{~mm}$ \\
$\mathrm{~N}_{\mathrm{rad}}$ & $1.072 \mathrm{X}_{0}$ \\
\hline \hline
\end{tabular}

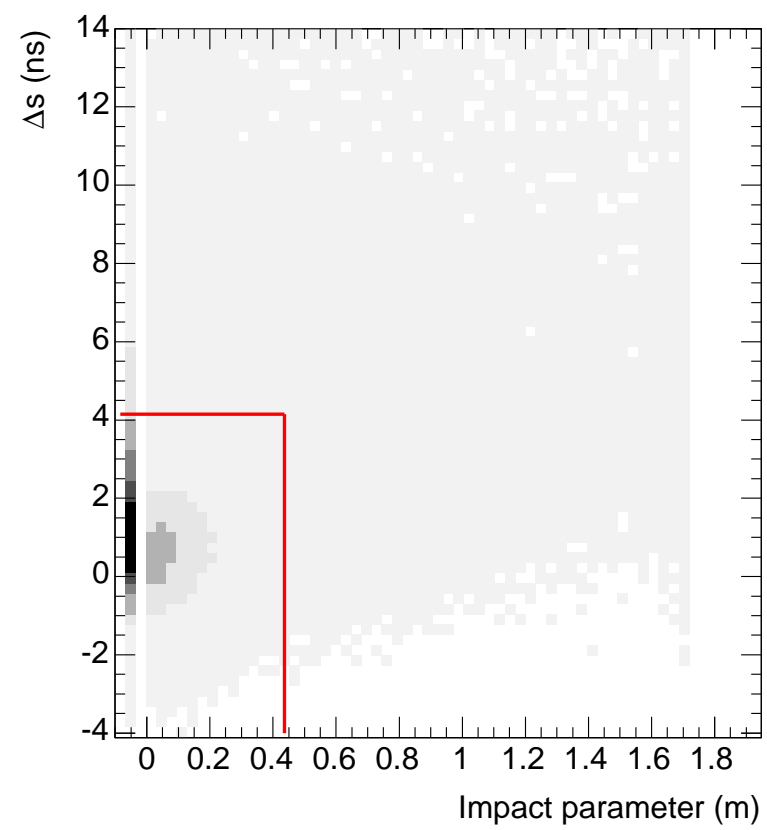

Figure 20: The relationship between $\Delta s$ and the impact parameter $b$ of a photon from $\tilde{\chi}_{1}^{0} \rightarrow \gamma \tilde{G}$ decays in a GMSB model with $m_{\tilde{\chi}}=70 \mathrm{GeV} / c^{2}$ and $\tau_{\tilde{\chi}}=10 \mathrm{~ns}$. We show the selection requirements that give us the smallest $95 \%$ C.L. cross section limit in a $\gamma+\not_{T}+$ jets analysis. The photons without impact parameter measurement are assigned a $b=-0.1 \mathrm{~m}$. Due to the low cut on the impact parameter there are about as many events in the low- $\Delta s$ high- $b$ as in the high- $\Delta s$ low- $b$ quarter, leading to a similar efficiency for a pure $b$-cut compared to a pure $\Delta s$ cut. The combined restriction leads to improved signal sensitivity. 


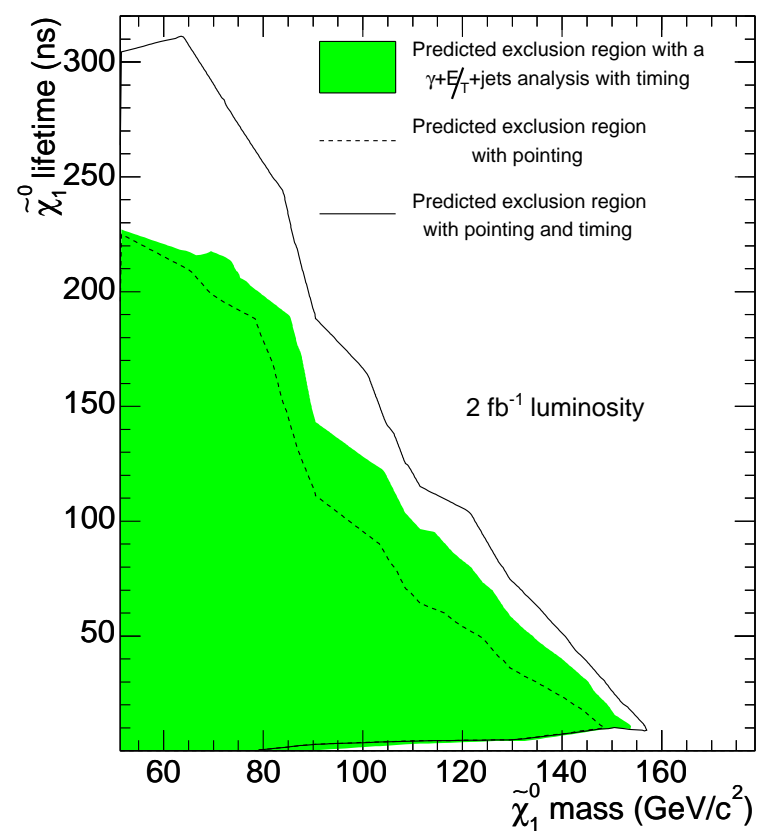

Figure 21: A comparison of the expected exclusion regions as a function of neutralino mass and lifetime for the GMSB model at $2 \mathrm{fb}^{-1}$ luminosity for a $\gamma+\not_{T}+$ jets analysis with photon pointing and timing. While timing generally yields a higher sensitivity than pointing, both methods would, if we combined them, extend the exclusion region further than either of them alone.

[1] CDF II Collaboration, F. Abe et. al., FERMILAB-Pub96/390-E.

[2] S. Kuhlmann et. al., Nucl. Instrum. Methods Phys. Res. A 518, 39 (2004); CDF Collaboration, P. T. Lukens, FERMILAB-TM-2198; for more details see http://hepr8.physics.tamu.edu/hep/emtiming/

[3] See for example CDF Collaboration, F. Abe et. al., Phys. Rev. D 59092002 (1999) and Phys. Rev. Lett. 81, 1791 (1998).

[4] Note that in principle the system can be used in conjunction with the Time of Flight system to search for charged particles as well. This becomes especially interesting for events in which the charged particle decay in flight and no track is reconstructed.

[5] See for example S. Ambrosanio et. al., Phys. Rev. D 54, 5395 (1996); C. H. Chen and J. F. Gunion, Phys. Rev. D 58, 075005 (1998).

[6] At CDF $x_{f}$ is measured by the shower-maximum detector $(\mathrm{CES} / \mathrm{PES})$ in the electromagnetic calorimeter and $x_{i}$ is the collision point, measured by the Silicon Vertex Chamber (SVX). The $t_{i}$ is calculated by the Central Outer Tracker (COT) and the Time of Flight (TOF) system which use the momentum and the measured time of flight of the charged particles of the underlying event emerging from the collision point and $t_{f}$ is the time of arrival of the photon from the EMTiming system.

[7] M. Goncharov, priv. comm.

[8] DØ Collaboration, S. Abachi et. al., Nucl. Instr. Methods Phys. Res. A 338, 185 (1994).

[9] DØ Collaboration S. Abachi et. al. Phys. Rev. Lett. 78, 2070 (1997), and B. Abbott et. al. Phys. Rev. Lett. 80, 442 (1998).

[10] CDF Collaboration, D. Acosta et. al., Phys. Rev. Lett. 89, 281801 (2002).

[11] DØ Collaboration, B. Abbott et. al., Phys. Rev. Lett. 82, 29 (1999).

[12] Note that we do not assume that any analysis without EMTiming at CDF is robust enough to search for longlived particles since there are no current collider experiments which have results without an additional handle such as photon pointing as done at LEP or DØ. For a discussion of photon pointing see for example ALEPH Collaboration, D. Decamp et. al., Nucl. Instrum. Methods Phys. Res. A 294, 121 (1998) and D. Cutts and G. Landsberg, arXiv:hep-ph/9904396

[13] ALEPH Collaboration, A. Heister et. al., Eur. Phys. J. C 25, 339 (2002); A. Garcia-Bellido, Ph.D. thesis, Royal Holloway University of London, 2002 (unpublished), 
arXiv:hep-ex/0212024

[14] See for example K. Olive, arXiv:hep-ph/9911307 and references therein.

[15] We follow B. C. Allanach et. al., Eur. Phys. J. C25, 113 (2002), and take the messenger mass scale $M_{\mathrm{M}}=2 \Lambda$, $\tan (\beta)=15, \operatorname{sgn}(\mu)=1$ and the number of messenger fields $N_{\mathrm{M}}=1$; $c_{\text {Grav }}$ (gravitino mass factor) and $\Lambda$ (supersymmetry breaking scale) unrestricted.

[16] T. Sjöstrand, L. Lönnblad and S. Mrenna, arXiv:hep-ph/0108264 We used version 6.158.

[17] F. Paige and S. Protopopescu, BNL Report BNL38034, 1986; F. Paige, S. Protopopescu, H. Baer and X. Tata, hep-ph/0001086 We use version 7.64 to generate the SUSY masses.

[18] J. Conway, http://www.physics.rutgers.edu/jconway/ research/software/pgs/pgs.html. We used version 3.21.

[19] Note that we have chosen a neutralino mass of $70 \mathrm{GeV} / c^{2}$ as an example, where the boost of the neutralino is lowest, to better illustrate the separation between single and diphoton case. When we present cross section limits we move to a mass of $110 \mathrm{GeV} / c^{2}$ where timing provides sensitivity outside the current GMSB exclusion region from LEP II.
[20] E. Boos, A. Vologdin, D. Toback and J. Gaspard, Phys. Rev. D 66, 013011 (2002).

[21] Following Ref. 3] we have neglected the contribution from cosmic ray backgrounds. The probability of an event with a real photon and a fake photon from a cosmic ray is small, and can be removed with simple topology cuts with very high efficiency. Similar arguments hold for the even more unlikely case of two fake photons from cosmic ray background sources.

[22] Following [11] we have neglected the contribution from cosmic ray backgrounds. The probability of an event with two jets overlapping with a photon from a cosmic ray is small, and and might be removed by requiring events where the photon is equal in magnitude and opposite in $\phi$ of the $\not_{T}$.

[23] R. Culbertson et. al., hep-ph/0007080 (especially Fig. 18 therein).

[24] L. Balka et. al., Nucl. Instr. Methods Phys. Res. A 267, 272 (1988); CDF Collaboration, F. Abe et. al., Phys. Rev. Lett. 73, 2662 (1994); D. Partos, Ph.D. thesis, Brandeis University, 2001, FERMILAB-THESIS-200121. 